\title{
Benchmark for $A b$ Initio Prediction of Magnetic Structures Based on Cluster-Multipole Theory
}

\author{
M.-T. Huebsch $\odot,{ }^{1,2}$ T. Nomoto $\odot{ }^{2}$ M.-T. Suzuki $\odot,{ }^{3,4}$ and R. Arita $\odot^{1,2}$ \\ ${ }^{1}$ Center for Emergent Matter Science, RIKEN, Wako, Saitama 351-0198, Japan \\ ${ }^{2}$ University of Tokyo, 7-3-1 Hongo, Bunkyo-ku, Tokyo 113-8656, Japan \\ ${ }^{3}$ Center for Computational Material Science, Institute for Materials Research, \\ Tohoku University, Sendai, Miyagi 980-8577, Japan \\ ${ }^{4}$ Center for Spintronics Research Network, Graduate School of Engineering Science, \\ Osaka University, Toyonaka, Osaka 560-8531, Japan
}

(Received 1 September 2020; revised 17 November 2020; accepted 11 January 2021; published 16 February 2021)

\begin{abstract}
The cluster-multipole (CMP) expansion for magnetic structures provides a scheme to systematically generate candidate magnetic structures specifically including noncollinear magnetic configurations adapted to the crystal symmetry of a given material. A comparison with the experimental data collected on MAGNDATA shows that the most stable magnetic configurations in nature are linear combinations of only few CMPs. Furthermore, a high-throughput calculation for all candidate magnetic structures is performed in the framework of spin-density functional theory (SDFT). We benchmark the predictive power of CMP + SDFT with 2935 calculations, which show that (i) the CMP expansion administers an exhaustive list of candidate magnetic structures, (ii) CMP + SDFT can narrow down the possible magnetic configurations to a handful of computed configurations, and (iii) SDFT reproduces the experimental magnetic configurations with an accuracy of $\pm 0.5 \mu_{B}$. For a subset the impact of on-site Coulomb repulsion $U$ is investigated by means of $1545 \mathrm{CMP}+\mathrm{SDFT}+U$ calculations revealing no further improvement on the predictive power.
\end{abstract}

DOI: 10.1103/PhysRevX.11.011031

\section{INTRODUCTION}

The grand challenge in first-principles calculation for magnetic materials is whether we can predict the experimental magnetic structure for a given material. Among a variety of possible functional materials, noncollinear magnets are a fascinating playground for materials design $[1,2]$, as they facilitate a wide range of fundamental phenomena and possible applications.

For example, in the context of antiferromagnetic (AFM) spintronics [3] there is a particular interest in noncollinear antiferromagnetism sparked by (i) its robustness against perturbations due to magnetic fields, (ii) a quasiabsence of magnetic stray fields disturbing, for instance, nearby electronic devices, and (iii) ultrafast dynamics of AFM domain walls [4], as well as (iv) its ability to generate large magnetotransport effects [5-7]. Hence, the optimization of AFM materials would open the door for applications such as seamless and low-maintenance energy generation,

Published by the American Physical Society under the terms of the Creative Commons Attribution 4.0 International license. Further distribution of this work must maintain attribution to the author(s) and the published article's title, journal citation, and DOI.
Subject Areas: Computational Physics, Magnetism, Materials Science ultrafast spintronics, and robust data retention, as well as be a guide toward advancing fundamental understanding of magnetotransport.

However, first-principles calculations with the generalized gradient approximation (GGA) in the framework of spin-density functional theory (SDFT) for magnetic materials have a problem: It is still an open question how accurately SDFT GGA can reproduce the experimental magnetic ground state. While SDFT has been widely used in studies on various magnets [8], there has been no systematic benchmark calculation for noncollinear AFM materials. Previous attempts have been restricted to collinear magnetism [9] or even stricter symmetry constrains [10-12]. In regard to noncollinear AFM materials, highthroughput calculations have been limited to setting the experimentally determined magnetic configuration as an initial guess [13]. A recently proposed attempt to predict magnetic structures based on a genetic evolution algorithm [14] strongly relies on the proper prediction of the magnetic ground state by SDFT. The lack of a systematic benchmark calculation is a consequence of the fact that it is a highly nontrivial task to investigate all the local minima in the SDFT energy landscape. Indeed, to search for all the (meta)stable states, we need an exhaustive list of physically reasonable magnetic configurations for which first-principles calculations can be performed. 
To this end, we devise the so-called cluster-multipole (CMP) expansion, which enables the expansion of an arbitrary magnetic configuration in terms of an orthogonal basis set of magnetic multipole configurations. By means of the CMP expansion, a list of initial magnetic structures for self-consistent GGA calculations is efficiently and systematically generated. With this at hand, a systematic high-throughput calculation with 2935 calculations has been performed.

The structure of the paper is as follows: In Sec. II, we explain the basic idea of CMP (Sec. II A) and setup for GGA calculation (Sec. II B). In Sec. III, the magnetic configuration of 131 materials is predicted using a combination of the CMP expansion and SDFT (CMP + SDFT). A comparison to the experimental data shows that the magnetic ground state can be narrowed down to be among a handful of computed configurations and SDFT reproduces the experimental on-site magnetic moment with an accuracy of approximately $\pm 0.5 \mu_{B}$. This benchmark, which is summarized in Sec. IV, thus provides a solid foundation for the $a b$ initio predictions of various magnetic properties.

\section{METHODS}

In this section, we briefly discuss the employed methods, namely the CMP expansion and SDFT. As the CMP expansion is a rather novel approach [15-17], it shall be motivated and set out in some detail. However, for more background and details of the algorithm, see Ref. [18]. SDFT, on the other hand, is a well-established method $[8,19]$. It is available as part of many ab initio packages [20-25] in its generalized version [26,27], which is applicable to noncollinear AFM configurations. Here, we chose to use the Vienna ab initio simulation package VASP $[20,28,29]$ and, hence, we merely elaborate on the setup details employed in this study.

\section{A. Cluster-multipole expansion}

The cluster-multipole expansion for magnetic structures $[15,18]$ provides an orthogonal basis set of magnetic configurations, which are symmetrized based on the crystallographic point group. In order to motivate the expansion, let us consider the vector Poisson equation:

$$
\nabla^{2} \boldsymbol{A}(\boldsymbol{r})=-\frac{4 \pi}{c} \boldsymbol{j}(\boldsymbol{r})
$$

where $\boldsymbol{j}(\boldsymbol{r})=c \nabla \times \boldsymbol{M}(\boldsymbol{r})$ is the current density and $\boldsymbol{M}(\boldsymbol{r})$ is the magnetization density. Here, the Coulomb gauge $\nabla \cdot \boldsymbol{A}(\boldsymbol{r})=0$ is invoked and the potential outside of the magnetization density is considered. The rotational invariance of $\nabla^{2}$ allows the vector gauge potential $\boldsymbol{A}(\boldsymbol{r})$ to be expanded with respect to vector spherical harmonics $\boldsymbol{Y}_{p q}^{l 1}$ [30]. Accordingly, the magnetic field $\boldsymbol{B}(\boldsymbol{r})=\boldsymbol{\nabla} \times \boldsymbol{A}(\boldsymbol{r})$ can be written in terms of magnetic multipole moments $M_{\mathrm{lm}}$ as follows [31]:

$\boldsymbol{B}(\boldsymbol{r})=-\sum_{l=1}^{\infty} \sum_{m=-l}^{l} \sqrt{4 \pi(l+1)} M_{\mathrm{lm}} \frac{1}{r^{l+2}} \boldsymbol{Y}_{\mathrm{lm}}^{l+1,1}(\Omega)$,

where $l$ is the orbital angular momentum quantum number and $m$ the magnetic quantum number.

Following Suzuki et al. in Ref. [18] the CMPs for a magnetic configuration on a point form $|m\rangle=$ $\left(\boldsymbol{m}_{1}, \boldsymbol{m}_{2}, \ldots, \boldsymbol{m}_{N}\right)^{T}$ read

$$
M_{\mathrm{lm}}=\sqrt{\frac{4 \pi}{2 l+1}} \sum_{i=1}^{N} \boldsymbol{m}_{i} \cdot\left[\nabla\left(\left|\boldsymbol{r}_{i}\right|^{l} Y_{m}^{l *}\right)\right] .
$$

$\boldsymbol{m}_{i}$ is a local magnetic moment on the magnetic site $i$ at position $\boldsymbol{r}_{i}$. For a given point group the point form is a set of all symmetrically equivalent points and can be classified into Wyckoff positions [32] in analogy to the Wyckoff positions of space groups. Here, $N$ is the multiplicity of the Wyckoff position of the point form, that constitutes the magnetic configuration. As introduced by Ref. [18], a point form carrying a magnetic configuration is referred to as (magnetic) cluster in the context of the CMP expansion for magnetic structures. In contrast to Ref. [18], here we do not introduce toroidal moments.

Symmetrization according to irreducible representations of the crystallographic point group allows for a physically meaningful expansion with respect to symmetrized harmonics,

$$
\mathcal{Y}_{l \Gamma \gamma}(\Omega)=\sum_{m=-l}^{l} c_{\operatorname{lm}}^{(\Gamma \gamma)} Y_{\operatorname{lm}}(\Omega),
$$

where $\Gamma$ indicates the irreducible representation and $\gamma$ the existing components of $\Gamma$. Here, the tabulated coefficients [30] $c_{\operatorname{lm}}^{\gamma}$ are chosen to be real valued. With this a virtual cluster [18] is constructed, where each magnetic site is assigned a magnetic moment. By mapping $l \gamma \rightarrow n$ through a Gram-Schmidt orthonormalization scheme, the CMP basis is computed.

The CMP basis can be written as

$$
\left\{|n\rangle=\left(\boldsymbol{e}_{1}^{(n)}, \boldsymbol{e}_{2}^{(n)}, \ldots, \boldsymbol{e}_{N}^{(n)}\right)^{T}\right\},
$$

where $\boldsymbol{e}_{i}^{(n)}$ is a unit vector of a local magnetic moment on the magnetic site $i$. By convention $n=1,2,3$ corresponds to ferromagnetism, while $n \geq 4$ corresponds to more complicated higher-order magnetic configurations including noncollinear magnetism. The definition of $|n\rangle$ coincides with $\left\{\boldsymbol{e}_{l \gamma}^{\mu}\right\}$ in Ref. [18] up to the choice of normalization [33].

In case that the period of the magnetic order coincides with that of the crystal structure, the propagation vector of 
the magnetic order $\boldsymbol{q}$ is zero. The magnetic structure is said to exhibit $\boldsymbol{q}=\mathbf{0}$ magnetism. Note that 3 continuous degrees of freedom of rotation of the magnetic moment per magnetic site for a total of $N$ magnetic sites yields $3 N$ linearly independent magnetic configurations and thus $n=1, \ldots, 3 N$. In this work, the configuration space of $\boldsymbol{q}=\mathbf{0}$ magnetic structures is explored.

The CMP basis defined in Eq. (5) is complete,

$$
\frac{1}{N} \sum_{n=1}^{3 N}|n\rangle\langle n|=\mathbb{1}_{3 N \times 3 N},
$$

and obeys the orthogonality relation:

$$
\left\langle n \mid n^{\prime}\right\rangle=N \delta_{n n^{\prime}} .
$$

Finally, the symmetry-adapted CMP coefficient reads

$$
M_{n}=\sum_{i=1}^{N} \mathbf{m}_{i} \cdot \mathbf{e}_{i}^{(n)}=\langle m \mid n\rangle=\langle n \mid m\rangle .
$$

In case of more than one inequivalent site exhibiting a magnetic moment, the space of all possible magnetic configurations is spanned by

$$
\left\{\left|n_{c_{1}}\right\rangle \otimes\left|n_{c_{2}}\right\rangle \otimes \cdots \otimes\left|n_{c_{d}}\right\rangle\right\},
$$

where $d$ is the number of clusters. Based on the above, an arbitrary magnetic configuration can be expanded as

$$
\begin{gathered}
|m\rangle=\left|m_{c_{1}}\right\rangle \otimes\left|m_{c_{2}}\right\rangle \otimes \cdots \otimes\left|m_{c_{d}}\right\rangle, \\
\left|m_{c_{j}}\right\rangle=\frac{1}{N^{\left(c_{j}\right)}} \sum_{n=1}^{3 N^{\left(c_{j}\right)}} M_{n}^{\left(c_{j}\right)}\left|n_{c_{j}}\right\rangle .
\end{gathered}
$$

Any two magnetic configurations on the same magnetic sites can be compared by an overlap, which we define as

$$
\mathcal{O}_{m m^{\prime}}=\left(\frac{\left\langle m \mid m^{\prime}\right\rangle}{\sqrt{\langle m \mid m\rangle} \sqrt{\left\langle m^{\prime} \mid m^{\prime}\right\rangle}}\right)^{2} .
$$

Lastly, notice that each CMP carries a definite order and irreducible representation (irrep). Additionally, CMPs of same order and irrep can be enumerated by a label $y$. This is a convention to write, for instance, $\left|n\left(6 T_{2 u} ; y\right)\right\rangle$, where the CMP labeled $n$ is the $y$ th CMP of sixth order and irrep $T_{2 u}$. We recall that the sixth order multipole is called 64-pole in the $2^{l}$ nomenclature.

\section{B. Setup for SDFT}

The $a b$ initio calculations are performed by VASP in version $5.4[20,28,29]$ and the flags are set appropriate to noncollinear SDFT GGA calculation including spin-orbit coupling as described in the Supplemental Material [34]. The pseudopotentials were chosen such that $d$ electrons in transition metals and $f$ electrons in lanthanoids and actinoids are treated as valence electrons. The default exchange-correlation functional, i.e., the generalized gradient approximation [35] by Perdew, Burke, and Ernzerhof (PBE), is used.

The VASP input is created by the aid of the PYTHON Materials Genomics (PYMATGEN) package [36]. In particular, we used subroutines based on SPGLIB [37]. The magnetic configurations of the CMP basis are created by a code authored by Suzuki, which employs the TSPACE library [38].

\section{RESULTS AND DISCUSSION}

In this section, we want to explore the following two main aspects.

(i) Is the CMP expansion a physically meaningful description of magnetic configurations? Namely, here the premise for a physically meaningful description constitutes that naturally occurring magnetic configurations can be characterized by one or few symmetrically related CMPs. It can be understood in the same sense as atomic orbitals are a meaningful basis to describe electrons bound to a free atom; i.e., the probability distribution of one electron is described by one or few degenerate atomic orbitals. In fact, this analogy extends to molecular orbitals in a complex, where the underlying spherical harmonics are symmetrized according to site symmetry.

(ii) Can SDFT predict the most stable magnetic configuration by the aid of an exhaustive list of candidate magnetic configurations for a given crystal? In fact, the predictive power of the combination of the CMP expansion and SDFT (CMP + SDFT) ought to be seen as a composition of the following issues.

(a) Is there evidence to assume that the list of candidate magnetic configurations generated by the CMP basis is exhaustive?

(b) Can the experimentally determined magnetic configuration be found among all SDFT results? Note that the similarity between two magnetic configurations is expressed by the overlap defined in Eq. (12). In addition, we compare the magnetic space group (MSPG), which crucially influences physical properties.

(c) Can SDFT correctly assign the lowest total energy to the experimental magnetic configuration?

\section{A. Investigated materials}

After preluding these questions, let us start by focusing on the experimental data found on MAGNDATA [39]. This 
(a) CMP expansion of experimental magnetic configurations

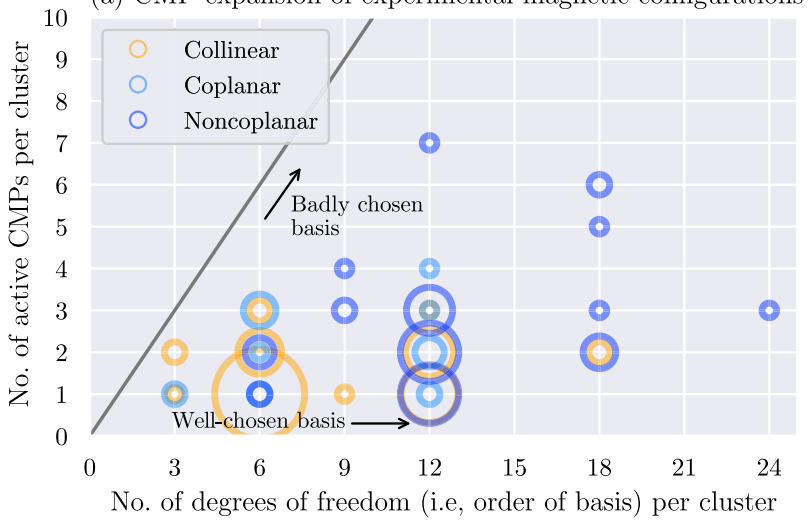

(b) Orbital character

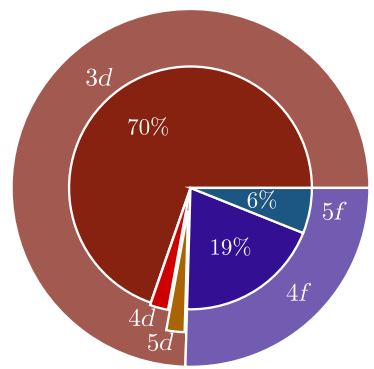

(c) Crystal system

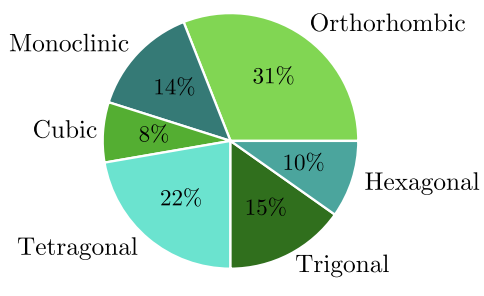

FIG. 1. Properties of 122 experimental magnetic configurations. (a) The number of CMPs needed to expand the experimental magnetic configuration - active CMPs - over the number of degrees of freedom per magnetic cluster. There are $3 \mathrm{~N}$ degrees of freedom for $N$ sites in a magnetic cluster, which coincides with the order of the CMP basis. The size of the circle indicates the frequency of occurrence. (b) Orbital character of the magnetic site. (c) Crystal system.

commendable collection of meticulously gathered neutron diffraction measurements and other measurements, e.g., optomagnetic response, is still growing and is by no means complete. The MAGNDATA entries used in this study were personally double-checked with the experimental references [40-160], and the specific compounds are listed in the Supplemental Material [34].

These materials explicitly contain transition metals, lanthanoids, and actinoids with on-site magnetic moments and most data entries are fully AFM or show only weak ferromagnetism. The magnetic configurations considered here possess zero propagation vector, which limits the available data to about 400 entries in MAGNDATA. Moreover, entries corresponding to duplicates in respect to higher temperature, pressure, or external magnetic field phases are excluded from this study. Finally, some large unit cells are omitted for efficiency reasons.

The still evolving nature of this database inclined us to take a differentiated perspective on each entry: For some materials the size of the magnetic moment is well determined, while the magnetic order could not be uniquely identified. And conversely, some materials have a wellknown symmetry, despite the lack of an exactly determined size of the magnetic moment. Therefore, in this study a total of 131 materials are analyzed, albeit they are distinguished in 122 entries with known magnetic order and 116 entries with known on-site magnetic moment.

Figure 1(a) presents the number of CMPs needed to describe a magnetic cluster featured in the experimental magnetic configuration over the total number of degrees of freedom in the corresponding magnetic cluster. Here, a nonzero CMP component is a so-called active CMP in analogy to the terminology used with respect to irreducible representations. The number of degrees of freedom per cluster is naturally equivalent to the order of the CMP basis.

The data shown in Fig. 1(a) comprise 162 magnetic clusters in 122 materials, among which 69 are classified to be collinear, 53 are noncollinear. In particular, 10 are coplanar and 43 are noncoplanar, as indicated by the color of the circles. Meanwhile, the size of the circle indicates the rate of occurrence.

A well-chosen basis is able to express a configuration in terms of few nonzero components. In this regard, remarkably $48.77 \%$ of all clusters are characterized by a single active CMP. And only six clusters, i.e., $3.70 \%$ of the clusters in the experimental configurations, are linear combinations of more than three CMPs.

The construction of the CMP basis [18] might intuitively raise the expectation that the number of active CMPs per cluster for a collinear magnetic structure is equal or less than three. Nevertheless, that could not have been generally expected for the noncollinear case. This intuition is empirically confirmed in Fig. 1(a), where all collinear circles are, as expected, reported below three active CMPs. In the case of noncollinear magnetic configurations, on the other hand, $\leq 3$ contributing CMPs per cluster strongly suggests that the basis is particularly well chosen. Thus, the CMP expansion of experimental configurations in Fig. 1(a) establishes the CMP basis to be a particularly suitable basis.

The pie charts in Fig. 1 give an overview of the composition of all 131 materials. In particular, Fig. 1(b) shows the orbital character of the valence electrons on the magnetic site. The majority of the materials feature transition metals with emerging $d$-orbital magnetism, while a minority of $25 \%$ observe $f$-orbital magnetism. Secondly, the pie chart in Fig. 1(c) presents the underlying Bravais lattice and fortifies a balanced mixture comprising all lattice types.

\section{B. Workflow and heuristic rule}

After we have discussed the known experimental properties, let us move on to setting up a predictive scheme. In Fig. 2 the computational workflow is organized in four 
1. Input

- obtain experimental magnetic configuration from MAGNDATA as .mcif file

\section{Setup}

- perform CMP expansion using Fortran code authored by M.-T. Suzuki (uses TSPACE library)

- read experimental magnetic configurations and CMP basis configurations as pymatgen structure

- create list of initial candidate magnetic configurations incl. linear combinations of same CMP order and irreducible representation

- write VASP input

3. Calculation

- run GGA for noncollinear magnetic magnetism in VASP

4. Analysis

- read final converged magnetic configuration as pymatgen structure

- determine key quantities:

$$
\begin{aligned}
\text { - } & \text { CMP+SDFT local minima } \\
\text { - } & \text { compare total energy to obtain } \\
& \text { CMP+SDFT global minimum } \\
\text { - } & \text { domains } \\
\text { - } & \text { overlaps btw. initial, final, and experimen- } \\
& \text { tal magnetic configurations } \\
\text { - } & \text { magnetic space groups }
\end{aligned}
$$

FIG. 2. Computational workflow divided into four steps: input, setup, calculation, and analysis.

steps: input, setup, calculation, and analysis. The input is taken in the form of (magnetic) crystallographic information files [161] from the database MAGNDATA.

Step 2 in Fig. 2, the setup, includes reading the magnetic crystallographic information files, creating the list of candidate magnetic configurations, and writing the input files for VASP by the aid of PYMATGEN. Crucially, in this step the CMP basis is obtained as described in Sec. II A, which does not require the experimental magnetic configuration as an input, but merely the choice of magnetic clusters.

We presume the following heuristic rule holds.

The magnetic ground state favors either pure CMPs or linear combinations of CMPs that combine equally weighted CMPs of the same order and same irrep.

We may try to provide some physical intuition here, in an attempt to understand this heuristic rule. However, more importantly, we will present statistical evidence in the discussion of Figs. 4(b) and 4(c). Basically, Landau theory of second-order phase transitions [162] states that the change of the spin density $\delta n_{\sigma \sigma^{\prime}}^{(s)}$, that lowers the symmetry of the spin density $n_{0 \sigma \sigma^{\prime}}^{(s)}$ during a phase transition, can be expanded in basis functions $\phi_{\gamma}^{(\Gamma)}$ with physical irreps $\Gamma$ and corresponding components $\gamma$ :

$$
\begin{aligned}
& n_{\sigma \sigma^{\prime}}^{(s)}=n_{0 \sigma \sigma^{\prime}}^{(s)}+\delta n_{\sigma \sigma^{\prime}}^{(s)}, \\
& \delta n_{\sigma \sigma^{\prime}}^{(s)}=\sum_{\Gamma \gamma} \eta_{\gamma}^{(\Gamma)} \phi_{\gamma}^{(\Gamma)} .
\end{aligned}
$$

This introduces expansion coefficients $\eta_{\gamma}^{(\Gamma)}$, which are indeed connected to the order parameter $\eta^{(\Gamma)}$, i.e.,

$$
\eta^{(\Gamma) 2}=\sum_{\gamma} \eta_{\gamma}^{(\Gamma) 2}
$$

The total free energy $F$ can then be written in even powers of $\eta^{(\Gamma)}$ :

$$
F(p, T, \eta)=F_{0}(p, T)+\sum_{\Gamma} A^{(\Gamma)}(p, T) \eta^{(\Gamma) 2}+\mathcal{O}\left(\eta^{(\Gamma) 4}\right),
$$

where $p$ is pressure and $T$ is temperature. According to the standard Landau theory, there is one $\Gamma$ for which $A^{(\Gamma)}(p, T)$ must change sign and vanish at the transition point. Therefore, it is usually enough to consider the linear combination of components $\gamma$ within one irrep $\Gamma$ at least when $T$ or $p$ is close to the transition point. However, as a counterexample, within the lower symmetry phase far away from the transition point, mixing among components with different irreps may occur if no further symmetry breaking is induced.

The question remains of why we also expect CMPs of equal expansion order to be more likely to mix. First, we recall that the point group harmonics $\mathcal{Y}_{l \Gamma \gamma}(\Omega)$ defined in Eq. (4) are structured as linear combinations of spherical harmonics $Y_{\operatorname{lm}}(\Omega)$ of the same order $l$, such that they are transformed as irrep $\Gamma$ of a given point group. Thus, the CMP basis elements corresponding to a specific order $l$ and irrep $\Gamma$ always feature all components at that expansion order. Nevertheless, at different orders the same irrep might appear, which may cause the mixing of different order CMPs during the self-consistent calculations. Examples are materials where the magnetic structure has a large collinear contribution and a small tilt introducing noncollinearity.

Let us continue focusing on the computational workflow, that presents the core of our prediction scheme. The proposed heuristic rule prompts us to extend the list of initial candidate magnetic configurations by linear combinations of same order and same irrep. Neglecting linear 
combinations of pairs yields $(Y-1) Y$ additional guesses, for $Y$ being the number of CMPs with same order and same irrep.

In the case of more than one magnetic cluster, $d \geq 2$, this would lead to too many additional guesses. For the 73 materials of concern, where $d \geq 2$, we chose to combine only the exact same multipole projected onto a different magnetic cluster. In other words, the linear combination of CMPs with same order, same irrep, and same $y$ is taken, cf. the last paragraph of Sec. II A. Now this similarly leads to $(Y-1) Y$ additional guesses, but $Y$ is the number of CMPs, which are distinct only with respect to $c_{j}$.

Step 3 in Fig. 2, the VASP calculation, is performed as described in Sec. II B. The total number of SDFT calculations necessary is equal to the number of candidates. The list of candidates is composed of in total $\left(\sum_{j}^{d} 3 N^{\left(c_{j}\right)}\right) \mathrm{CMP}$ basis magnetic configurations and accordingly many times $(Y-1) Y$ additional guesses. This amounts to a total of 2935 calculations including all 131 materials in this study.

Step 4 in Fig. 2, the analysis, involves determining characteristic quantities of each calculation. First, all possible domains of the converged magnetic configuration are computed. To that end each space group operation combined with time reversal operations \pm 1 is applied, which leads to either (a) covering the magnetic configuration and thus the operation is element of the magnetic space group or (b) a new magnetic domain. Considering the set of operations that leave the magnetic configuration invariant, we determine the magnetic space group devising the identify magnetic group application on the Bilbao Crystallographic Server [163].

All calculations of a given material and their domains are cross-checked with each other in order to filter how many distinct magnetic configurations and, thus, distinct local minima in the SDFT total energy landscape have been identified. Quantities such as the total energy and the size of the magnetic moment per site are averaged over all calculation corresponding to the same local minimum. The calculation with the lowest total energy among all SDFT calculations of a given material is the CMP + SDFT global minimum.

Note that the list of candidates created as discussed in step 3 is not free of duplicates corresponding to different domains of the same magnetic configuration. In the Supplemental Material [34] the CMP basis for $\mathrm{YMnO}_{3}$ is constructed. Then, linear combinations of CMPs and magnetic domains are eluded by means of that example. The candidates corresponding to different domains could be excluded to avoid unnecessary numerical cost. This amounts to a total of 2313 unique calculations for all 131 materials in this study, which comprise of $35.75 \%$ additional guesses.

To conclude step 4 in Fig. 2, all possible domains are considered when computing the overlaps of (i) the experimental and the initial candidate's magnetic configuration,
$\mathcal{O}_{\text {expt,init }}$, (ii) the experimental and the converged SDFT calculation's final magnetic configuration, $\mathcal{O}_{\text {expt.fin, and }}$ (iii) the initial candidate's and the converged SDFT calculation's final magnetic configuration, $\mathcal{O}_{\text {fin,init }}$, as defined in Eq. (12).

In total this study identifies $2005 \mathrm{CMP}+$ SDFT local minima starting from 2313 unique candidates. As mentioned, we performed 2935 including some redundant candidates in this study. Instead of excluding these redundant candidates that correspond to different domains of the same magnetic configuration, we used them to statistically analyze the reproducibility. In a nutshell, the reproducibility is the probability to converge to the same local minimum, when repeating the SDFT calculation. More details are described in the Supplemental Material [34]. In this study the reproducibility reaches 0.79 on a scale from 0 to 1 , where 1 refers to perfect reproducibility.

\section{Performance of candidate magnetic configurations}

The high computational cost is justified, only if the list of candidates can be expected to be exhaustive. Let us recall that the CMP basis defined in Eq. (5) spans the space of all possible magnetic configurations. Each CMP is characterized by its order and irrep. First, we want to argue that the candidate's irrep is likely to prevail throughout the SDFT calculation. As the CMP basis is complete and, thus, any irrep that could be active in a given system explicitly appears in the CMP basis, the former corroborates that the CMP basis is a good starting point.

Figure 3(a) shows a histogram of the overlap of the final magnetic configuration and the initial candidate, $\mathcal{O}_{\text {fin,init }}$. In particular, $\mathcal{O}_{\text {fin,init }} \approx 1$ corresponds to the candidate's magnetic configuration remaining almost identical during the iterations. In that case, the candidate appears to be in close vicinity to a local minimum in the total energy landscape of SDFT. We see that the uppermost bin, with $46.54 \%$ of all calculations, accounts for more calculations than any other bin.

On the other hand, if the candidate does not correspond to a minimum in the total energy, the calculation is expected to yield a small overlap: $\mathcal{O}_{\text {fin,init }} \ll 1$. If the system converges to a magnetic configuration, which is a linear combination of the initial candidate and another magnetic configuration, a finite $\mathcal{O}_{\text {fin,init }}$ occurs.

There is a related scenario in which the system converges to a magnetic configuration that is of the same irrep, but does not include the CMP of the initial candidate. That case can be characterized by $\mathcal{O}_{\text {fin,init }} \approx 0$ and $\sigma_{\text {irrep }}=0$, warranted the definition of the variance of the irrep reads

$$
\sigma_{\text {irrep }}=\sum_{j, j^{\prime}}^{d} \sum_{n, n^{\prime}}^{3 N^{\left(c_{j}\right)}}\left|\hat{M}_{n}^{\left(c_{j}, \text { init }\right)}\right| B_{n n^{\prime}}\left|\hat{M}_{n^{\prime}}^{\left(c_{j}, \text { fin }\right)}\right|
$$



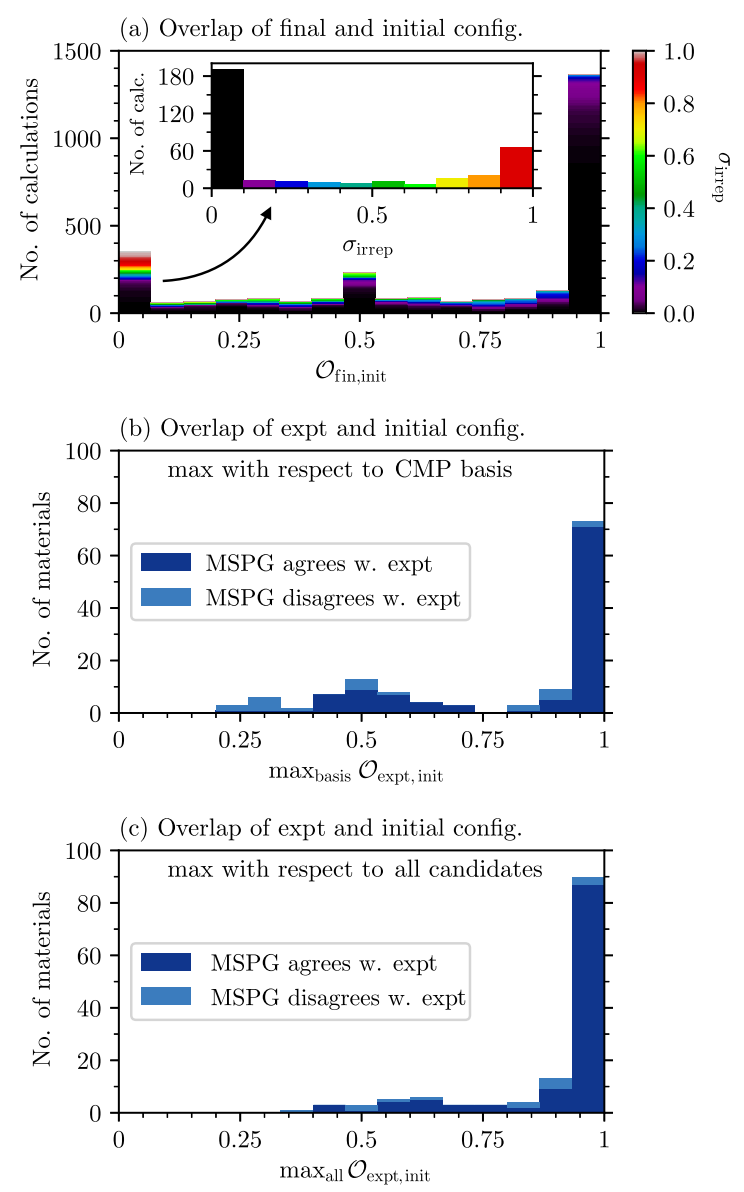

FIG. 3. (a) Overlap of the candidate and the final SDFT result. The overlap is defined in Eq. (12). The color scale indicates the variance of the irreducible representation. Inset: the variance of the irreducible representation for the lowermost bin (see arrow). (b) The maximum overlap of the experiment and the initial candidate with respect to the CMP basis. (c) The maximum overlap of the experiment and the initial candidate with respect to all candidates including the CMP basis and additional guesses. The color classifies if the magnetic space group (MSPG) agrees with the experimentally determined MSPG.

$$
\begin{aligned}
\hat{M}_{n}^{\left(c_{j}, \text { init/fin }\right)} & =\frac{M_{n}^{\left(c_{j}, \text { init/fin }\right)}}{\sum_{j=1}^{d} \sum_{n^{\prime}=1}^{3 N^{\left(c_{j}\right)}}\left|M_{n^{\prime}}^{\left(c_{j}, \text { init/fin }\right)}\right|}, \\
B_{n n^{\prime}} & = \begin{cases}1 & \text { irrep }_{n} \neq \text { irrep }_{n^{\prime}} \\
0 & \text { irrep }_{n}=\text { irrep }_{n^{\prime}} .\end{cases}
\end{aligned}
$$

Here, $\sigma_{\text {irrep }}$ is defined such that, if the same irreps appear with the same weight in the candidate's CMP expansion and in the CMP expansion of the converged calculation, then $\sigma_{\text {irrep }}=0$. In a nutshell, $\left|\hat{M}_{n}\right|$ indicates to what percentage the $n$th CMP contributes to the expansion and $B_{n n^{\prime}}$ is a Boolean giving zero weight to equal irreps.

The color bar in Fig. 3(a) corresponds to $\sigma_{\text {irrep }}$ defined in Eq. (17). The variance of the irrep is less than $10 \%$, $\sigma_{\text {irrep }}<0.1$, in $78.16 \%$ of all SDFT calculations. In other words, the inital irrep is highly likely to be active in the final magnetic configuration.

The inset of Fig. 3(a) emphasizes this observation: The variance of the irrep for the lowermost bin of Fig. 3(a) is shown as a histogram. Notably, the initial irrep has less than $10 \%$ deviation, i.e., $\sigma_{\text {irrep }}<0.1$, in $53.95 \%$ of the calculations with $\mathcal{O}_{\text {fin,init }} \approx 0$.

As a more general statement, we have shown that the candidate's irrep is statistically likely to prevail throughout the SDFT calculation. Conversely, the most stable magnetic configuration is less likely to be found, if the irrep is not among the list of candidates. Hence, creating a list of candidates building upon the CMP basis is an efficient solution to assure all possible irreps are among the candidates.

From this point of view, it seems unnecessary to introduce additional guesses as candidates that are equally weighted CMPs of same order and same irrep. However, using the experimental data as a guide once more, the advantages of including additional guesses into the list of candidates becomes clear.

Figure 3(b) presents the maximum overlap of the CMP basis and the experiment, $\max _{\text {all }} \mathcal{O}_{\text {init,expt }}$. The histogram shows a probability density strongly peaked close to one. Additionally, there are side peaks at $1 / 3$ and $1 / 2$. This bias toward $1 / 3$ and $1 / 2$ can be appreciated when considering the aforementioned heuristic rule once again.

Namely, the magnetic ground state favors either pure CMPs or linear combinations of CMPs that combine equally weighted CMPs of the same order and same irrep. An irrep can have a dimension of 1,2, or 3 and accordingly at each CMP order CMP basis configurations occur in sets of 1,2 , or 3 configurations in the expansion. Hence, favored linear combinations projected onto a CMP basis configuration are prone to yield overlap of $1,1 / 2$, or $1 / 3$.

In comparison, Fig. 3(c) displays the maximum overlap of initial candidate and the experiment, $\max _{\text {all }} \mathcal{O}_{\text {init,expt }}$, with respect to the complete list of candidates, which contains the CMP basis configurations as well as additional guesses. The introduction of additional guesses, following the heuristic rule, can effectively avoid side peaks at $1 / 3$ and $1 / 2$ and thus takes into account linear combinations common in materials existing in nature.

As Fig. 3(a) shows, most magnetic configurations remain close to the initial magnetic configuration. Therefore it is paramount to start from an exhaustive list of magnetic configurations.

The dark blue and light blue colors in Figs. 3(b) and 3(c) indicate that the magnetic space group found experimentally is identical to the magnetic space group of the candidate or not, respectively. Considering all candidates, as in Fig. 3(c), 117 of 122 magnetic space groups agree. This is an improved agreement rate compared to considering only the CMP basis, as in Fig. 3(b), where 110 magnetic space groups agree. It is noteworthy that some 
magnetic space groups only enter the list of candidates through the additional guesses.

A final argument in favor of introducing additional guesses is that in total we find 655 of 2005, hence $32.67 \%$, of the local minima in the SDFT energy landscape only thanks to the additional guesses. Even among the CMP + SDFT minima with the minimum total energy 23 are thanks to the additional guesses, as well as 15 of the (local) minima most similar to the experiment.

Therefore, with the collection of arguments mentioned above, we have justified the expectation that the list of candidate magnetic configurations is exhaustive. In the following, we investigate whether the experimentally determined magnetic configuration is present among all SDFT results and how we might predict the likely experimental magnetic configuration for an unknown material.

\section{Analysis of CMP+SDFT local minima}

Following the workflow in Fig. 2 all final SDFT results are scrutinized for their similarity. Some SDFT results correspond to the same local minimum in the SDFT total energy landscape and as such they are grouped in $\mathrm{CMP}+$ SDFT local minima. The overlap of each CMP + SDFT local minimum with the experimental magnetic configuration is computed according to Eq. (12). The $\mathrm{CMP}+\mathrm{SDFT}$ minimum that yields the maximum overlap with the experiment $\max _{\{l \mathrm{~m}\}} \mathcal{O}_{\text {fin,expt }}$ (MAXOEXP) is termed to be the most similar CMP + SDFT local minimum to the experiment. A worthwhile run should yield $\max _{\{\operatorname{lm}\}} \mathcal{O}_{\text {fin,expt }} \approx 1$, entailing that MAXOEXP is indeed very similar to the experiment. Additionally, the magnetic space group should agree with the experimentally detected symmetry.

Figure 4(a) presents $\max _{\{\operatorname{lm}\}} \mathcal{O}_{\text {fin,expt }}$, i.e., the overlap of MAXOEXP for 122 materials with known experimental magnetic order. The distribution features a substantial peak at $\max _{\{\mathrm{Im}\}} \mathcal{O}_{\text {fin,expt }} \approx 1$. In fact, $82.44 \%$ of MAXOEXP $\operatorname{mark} \max _{\{\operatorname{lm}\}} \mathcal{O}_{\text {fin,expt }}>0.75$, verifying good agreement of one CMP + SDFT local minimum with the experiment.

Despite the large overlap, some MSPG do not agree. In particular $70.99 \%$ of MAXOEXP agree with respect to their MSPG, despite yielding $\max _{\{\mathrm{lm}\}} \mathcal{O}_{\text {fin,expt }}>0.75$; see the dark blue labeled "o" in Fig. 4(a).

The uppermost bin in Fig. 4(a) accumulates 54.96\% and corresponds to $\max _{\{\operatorname{lm}\}} \mathcal{O}_{\text {fin,expt }}>0.96$. Even in the uppermost bin not all MSPG agree, while on the other hand, most $\mathrm{CMP}+\mathrm{SDFT}$ local minima with rather inadmissible $\max _{\{\operatorname{lm}\}} \mathcal{O}_{\text {fin,expt }}<0.75$ still agree with respect to their MSPG. For instance, $\mathrm{Fe}_{2} \mathrm{O}_{3}$ has a collinear AFM structure with a small tilting [148]. While the parent SPG is $R \overline{3} c$ (167), the small tilting results in $P \overline{1}$ (2.4) for the MSPG. In the CPM expansion, the experimental configuration is described by two CMP basis configurations of order 5 . However, they do not observe the same irreducible (a) Overlap of MAXOEXP

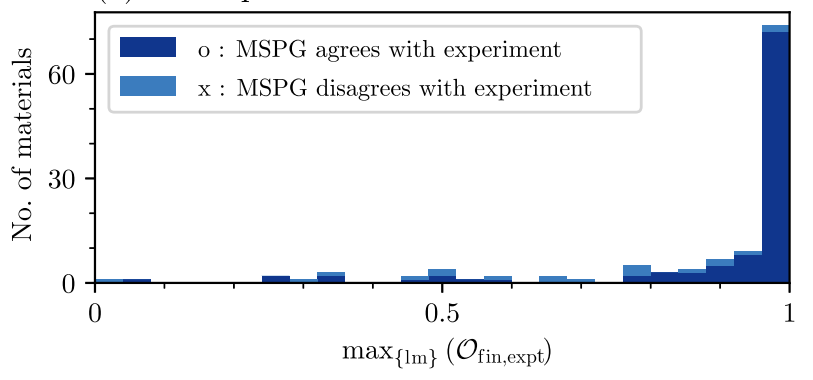

(b.1) $d$-orb. magnetism

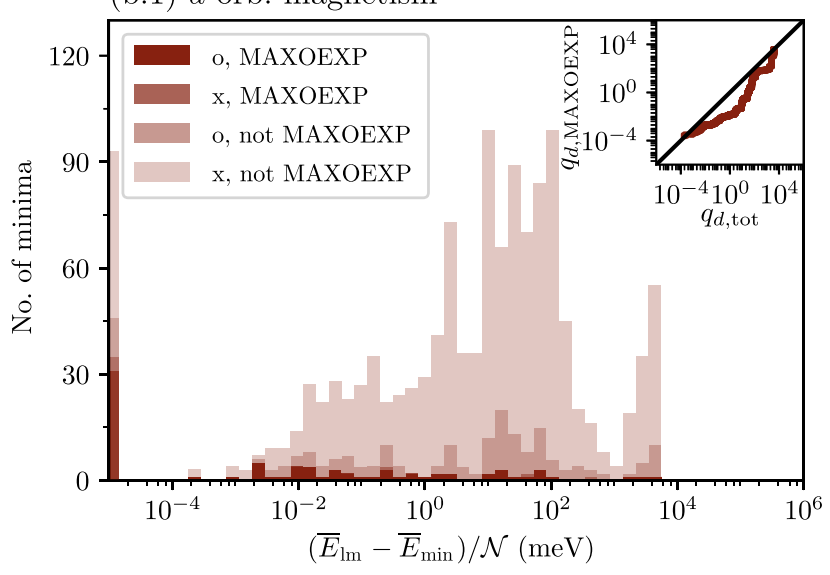

(b.2) $f$-orb. magnetism

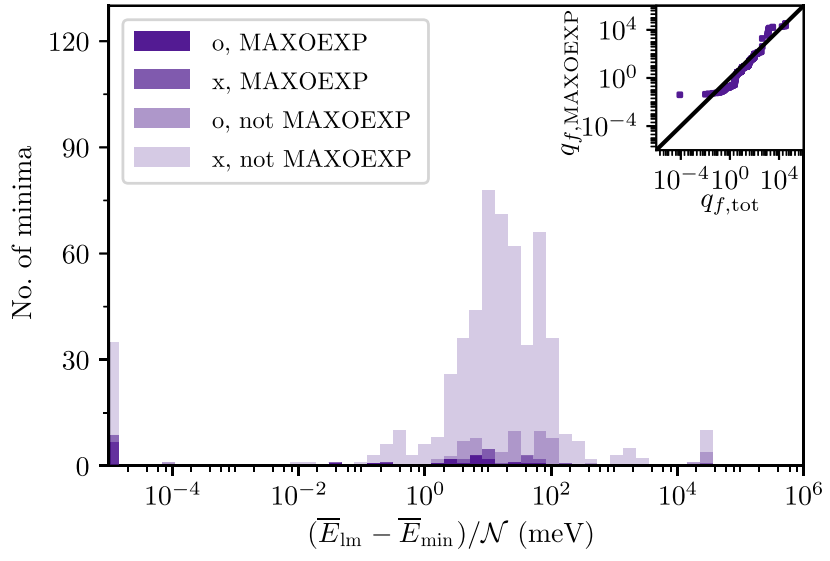

FIG. 4. (a) Overlap of experiment and CMP + SDFT minimum most similar to experiment (MAXOEXP). Overlap is defined in Eq. (12). o or $\mathrm{x}$ classifies if the magnetic space group (MSPG) agrees or disagrees with the experimental MSPG. (b.1) Total energy distribution with respect to materials feature $d$-orbital magnetism. The minima are classified in MAXOEXP and remainder "not" MAXOEXP, and MSPG agrees or disagrees. Inset: $Q-Q$ plot, where $q_{d, \text { MAXOEXP }}$ with respect to the distribution of MAXOEXP is compared to $q_{d \text { tot }}$ with respect to the distribution of all local minima of materials feature $d$-orbital magnetism. (b.2) Total energy distribution with respect to materials feature $f$-orbital magnetism. Inset: $Q-Q$ plot, where $q_{f, \text { MAXOEXP }}$ with respect to the distribution of MAXOEXP is compared to $q_{f, \text { tot }}$ with respect to the distribution of all local minima of materials featuring $f$-orbital magnetism. 
representation. In particular, the main contribution is $A_{1 g}$ and the tilting is due to contributions of $E_{u}$. In CMP + SDFT the most stable configuration is pure $A_{1 g}$ without any tilting. So that, although the overlap $\max _{\{\operatorname{lm}\}} \mathcal{O}_{\text {fin,expt }}=$ 0.9658 , the MSPG predicted by CMP + SDFT is $R \overline{3} c$ (167.103) not $P \overline{1}$ (2.4) as found experimentally.

In total, $84.43 \%$ among MAXOEXP yield the correct MSPG. This is to say that neither the overlap nor the MSPG alone are a sufficient criterion whether the experimental configuration is correctly predicted or not.

In comparison, only $16.17 \%$ of all CMP + SDFT minima yield the experimental MSPG. However, for $90.16 \%$ of the materials at least one CMP + SDFT minima yields the experimental MSPG. As mentioned among MAXOEXP $84.43 \%$ yield the experimental MSPG.

Another characteristic CMP + SDFT minimum is the CMP + SDFT global minimum, which observes the minimum total energy in SDFT. Among all CMP + SDFT global minima only $37.70 \%$ yield the experimental MSPG. This shows that the MSPG of the CMP + SDFT global minima is more likely to agree with the experimental MSPG than a random CMP + SDFT minimum, but the $\mathrm{CMP}+\mathrm{SDFT}$ global minima is not adequately predicting the MSPG.

Let us continue by analyzing the SDFT total energy of the CMP + SDFT minima in more detail. Each CMP + SDFT minimum is attributed one or more SDFT results, as multiple candidates might converge to the same minimum. An average over these attributed SDFT results leads to the material dependent and magnetic configuration dependent total energy of a specific CMP + SDFT minimum $\bar{E}_{\mathrm{lm}}$. The $\mathrm{CMP}+\mathrm{SDFT}$ global minimum observes the minimum total energy $\bar{E}_{\text {min }}$.

In order to compare the total energy across materials, we take a normalized relative total energy that reads

$$
\left(\bar{E}_{\operatorname{lm}}-\bar{E}_{\min }\right) / \mathcal{N} \text {. }
$$

Here, $\mathcal{N}$ is the total number of degrees of freedom, i.e., the sum of the order of basis over all clusters that observe a magnetic moment in SDFT in that material.

Figures 4(b.1) and 4(b.2) present the distribution of CMP + SDFT minima over the normalized relative total energy of materials featuring $d$-orbital magnetism and $f$-orbital magnetism, respectively. The energy scale is logarithmic in units of $\mathrm{meV}$. And the lowermost bin, representing the CMP + SDFT global minima, would theoretically lie precisely at zero. However, for the obvious practical reasons, namely that $\log (0) \rightarrow-\infty$, it is added at the lower edge. The remaining bins represent the distribution of CMP + SDFT local minima $\rho_{d / f \text {,tot }}$. A key question is, whether MAXOEXP tends to be close to the total energy minimum.

In Figs. 4(b.1) and 4(b.2) the color intensity classifies all CMP + SDFT minima according to agreement or disagreement with the experimental MSPG labeled by o and $\mathrm{x}$, respectively. Additionally, the minima are classified according to being MAXOEXP or not. Overall, the total energy distributions $\rho_{d / f \text {,tot }}$ span across many orders of magnitude. Albeit, $\rho_{f \text {,tot }}$ is more concentrated in the energy range $1 \mathrm{meV}$ up to $1000 \mathrm{meV}$.

The data show that in total 43 of $122(35.25 \%)$ of the CMP + SDFT global minima coincide with MAXOEXP. Hence, the magnetic configuration with the minimum total energy in this study does not, at this point, identify the expected experimental configuration. Nevertheless, MAXOEXP might tend toward smaller total energy. In order to gain more insight, we ask if MAXOEXP data points follow the same distribution as an arbitrary local minimum in $\rho_{d / f, \text { tot }}$.

Two distributions can be compared in terms of a $Q-Q$ plot [164], where the $x$ axis represents the quantile of the reference distribution and the $y$ axis represents the quantile of the sample distribution. Let us define the quantile $q_{s / r}$ for a sample or reference distribution of local minima $\left\{\operatorname{lm}_{k}\right\}$, where $k=0, \ldots, K-1$, and the local minima (lm) are ordered by $\bar{E}_{\mathrm{lm}_{k}} \leq \bar{E}_{\mathrm{Im}_{k+1}}$. The $k /(K-1)$ quantile $q_{k}$ is given by

$$
q_{k}=\left(\bar{E}_{\operatorname{lm}_{k}}-\bar{E}_{\min }\right) / \mathcal{N} .
$$

Hence, the 0.5 quantile is simply the median value and the 0.1 quantile is the point that divides the distribution such that $90 \%$ of the local minima have greater total energy.

The inset of Fig. 4(b.1) shows the $Q-Q$ plot comparing quantiles of $\rho_{d, \text { MAXOEXP }}$ as the sample distribution, with $\rho_{d \text {,tot }}$ as the reference distribution. For each data point in the smaller sample distribution the quantile is computed, as explained above. Subsequently, $q_{d \text {,MAXOEXP }}$ is juxtaposed against $q_{d \text {,tot }}$.

If the two datasets are sampled from the same underlying distribution $\rho_{d, \text { MAXOEXP }}=\rho_{d \text {,tot }}$, all points align on the median. The quantile is defined on the same axis as the original distribution; i.e., $q_{d \text {,MAXOEXP }}$ and $q_{d \text {,tot }}$ are defined on $\left(\bar{E}_{\operatorname{lm}_{k}}-\bar{E}_{\min }\right) / \mathcal{N}$.

The $Q-Q$ plot in the inset of Fig. 4(b.1) shows significant deviation from the median. Indeed, the slow incline up to approximately $10 \mathrm{meV}$ reveals an accumulation of MAXOEXP toward lower total energy. For $d$-orbital magnetism we find 77.66\% of MAXOEXP below $1 \mathrm{meV}$. On average each material has 4.45 CMP + SDFT local minima below $1 \mathrm{meV}$. In particular, in this dataset the material with the maximum number of CMP + SDFT local minima has 18 minima below $1 \mathrm{meV}$. This shows that $\mathrm{CMP}+\mathrm{SDFT}$ successfully narrows down the possible magnetic configurations for a new material featuring $d$-orbital magnetism to a handful of CMP + SDFT local minima, that are highly likely to be close to the experimental observation. 
The inset of Fig. 4(b.2) shows the analogous $Q-Q$ plot for $f$-orbital magnetism. Here, the quantiles basically align on the median, suggesting that $\rho_{f, \operatorname{MAXOEXP}}=\rho_{f \text {,tot }}$. Moreover, for $f$-orbital magnetism we find only $32.43 \%$ of MAXOEXP below $1 \mathrm{meV}$. Although in case of $f$-orbital magnetism the consideration of the total energy seems to fail in narrowing down the number of possible magnetic configurations, at least the CMP + SDFT run itself proposes a set of 10-15 possible magnetic configurations.

The presented data opens a gateway to identifying a handful of magnetic configurations as CMP + SDFT local minima for a given material among which the experimentally stable magnetic space group and exact configuration is highly likely to be found. Yet it has not been possible to uniquely identify the ground state based on the SDFT total energy. Although CMP + SDFT yield local minima with the experimental MSPG and local minima with large overlap with the experimental magnetic configuration, SDFT fails to assign a low total energy compared to other local minima.

\section{E. Magnetic moment per site}

Besides the magnetic configuration, the size of the onsite magnetic moment crucially influences the magnetic properties of a material. Hence, it is interesting to ask if the magnetic moment estimated by SDFT is close to the experimentally determined magnetic moment per site. In the literature [165] it is well known that complexes containing first row transition metals with open $3 d$ orbitals are dominated by crystal field splitting. This is referred to as strong field regime. Further, the ground state of complexes containing lantanides with open $4 f$ orbitals are dominated by spin-orbit coupling. Complementary, this is referred to as weak field regime. Let us explore the implications by looking closer at the element dependence of the on-site magnetic moment.

Figure 5 presents the on-site magnetic moment averaged over sites within one magnetic cluster as a function of elements sorted by increasing number of electrons. In particular, the average magnetic moment per site reads

$$
\mu_{c_{j}}=\frac{1}{N^{\left(c_{j}\right)}} \sum_{i=1}^{N^{\left(c_{j}\right)}}\left|\boldsymbol{m}_{i}\right|,
$$

and, thus, the average is taken within each magnetic cluster $c_{j}$, only. The columns show the case of $3 d$-orbital magnetism and $4 f$-orbital magnetism, respectively.

Figure 5(a.1) gives an overview of the experimental results $\mu_{\text {expt }}$ for $3 d$-orbital magnetism. We see that within compounds featuring the same magnetic element vastly different on-site magnetic moments are reported. This is referred to as compound dependence in the following discussion. Overall, the maximum on-site magnetic moment per element frames a dome shape with a clear maximum at Mn closely followed by Fe. In comparison, Fig. 5(a.2) shows the on-site magnetic moment $\mu_{\text {th }}$ predicted by $\mathrm{CMP}+\mathrm{SDFT}$. Here, $\mu_{\mathrm{th}}$ is taken to be the magnetic moment of the magnetic configuration with MAXOEXP, which has the most similar magnetic order compared to the experiment. We can see very good agreement in the overall tendency between experiment and CMP + SDFT.

A strong crystal field represents a real and time reversal invariant perturbation that forces a real-valued ground state which effectively quenches the orbital angular moment operator $(\boldsymbol{L} \equiv \mathbf{0})$ as discussed in many textbooks; see, e.g., Ref. [166]. Therefore the spin contribution alone is expected to constitute the on-site magnetic moment. Fortunately, in contrast to the experiment the numeric calculation grants direct access to the spin contribution $\boldsymbol{\mu}_{s, \text { th }}$ and the angular momentum contribution $\boldsymbol{\mu}_{l, \mathrm{th}}$ to the on-site magnetic moment:

$$
\boldsymbol{\mu}_{\mathrm{th}}=\boldsymbol{\mu}_{s, \mathrm{th}}+\boldsymbol{\mu}_{l, \mathrm{th}} .
$$

Figure 5(a.3) presents the absolute values $\mu_{s, \mathrm{th}}$ and $\mu_{l, \mathrm{th}}$. The data clearly confirm that the angular momentum is almost entirely quenched in SDFT. Only for the heavier elements, where spin-orbit coupling becomes more relevant [167], a small contribution is given by $\mu_{l, \text { th }}$. In other words, SDFT supports that for compounds with more than halffilled $3 d$ bands, the angular momentum is only partially quenched.

The dominant $\mu_{s, \text { th }}$ can be directly compared to the spinonly magnetic moment in the ionic limit. It is computed within the Russel-Saunders coupling scheme and is given by

$$
\mu_{s, \text { ion }}^{(3 d)}=2 \sqrt{s(s+1)} \mu_{B},
$$

with spin quantum number $s$ for the total spin operator $S$. The total spin $S$ of the electronic configuration $3 d^{n}$ with $n$ electrons is essentially constructed by following Hund's first rules. Albeit in real complexes the electron configuration can be in the high spin (hs) or the low spin (ls) configuration depending on the crystal field strength compared to the intraorbital Coulomb repulsion. This yields different spinonly magnetic moments $\mu_{s, \text { ion }}^{(3 d)}$ in the ionic limit for electronic configurations of the form $3 d^{n}$ (hs or ls).

In Figure 5(a.3), $\mu_{s, \text { ion }}^{(3 d)}$ is displayed as a reference for various possible electronic configurations. Here, we assumed octahedral complexes for the crystal field splitting. The maximum magnetic moment is consistent with the experiment and CMP + SDFT calculation realized for $\mathrm{Mn}^{2+}$ or $\mathrm{Fe}^{3+}$ in the ionic limit. Additionally, the ionic limit already hints toward possible reasons for the observed compound dependence. Namely, we expect the formal oxidation state and the crystal field strength to introduce compound dependence. Further compound dependence arises due to the exact symmetry including small distortions as introduced by the Jahn-Teller effect and the choice of ligands via the nephelauxetic effect, which describes the 
(a.1) 3d-orb. magnetism: experiment

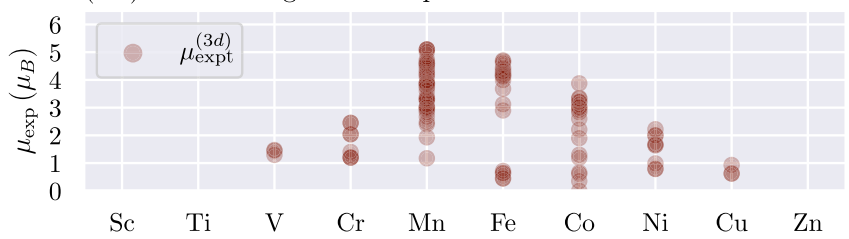

(a.2) 3d-orb. magnetism: CMP+SDFT

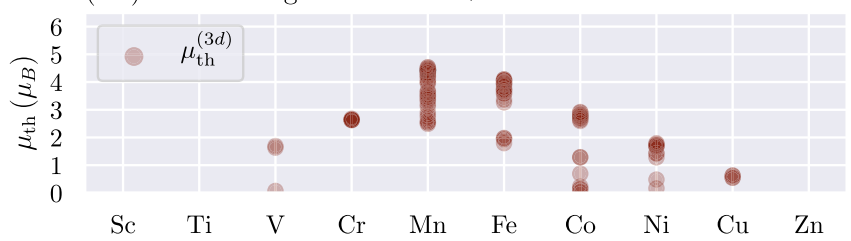

(a.3) Orbital and spin contributions of $\mu_{\mathrm{th}}^{(3 d)}$

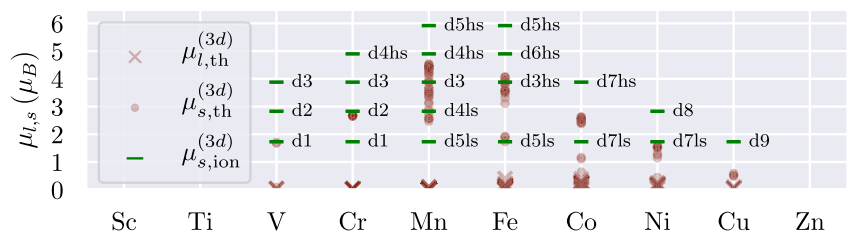

(b.1) $4 f$-orb. magnetism: experiment

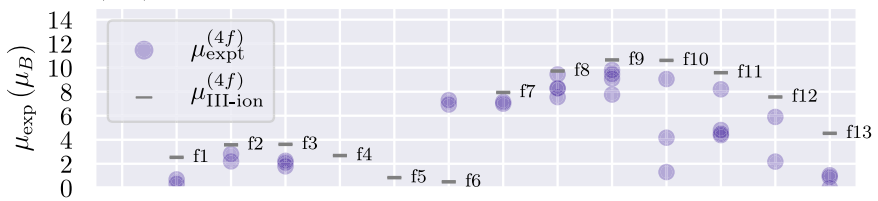

$\mathrm{La} \mathrm{Ce}$ Pr Nd Pm Sm Eu Gd Tb Dy Ho Er Tm Yb

(b.2) $4 f$-orb. magnetism: $\mathrm{CMP}+\mathrm{SDFT}$

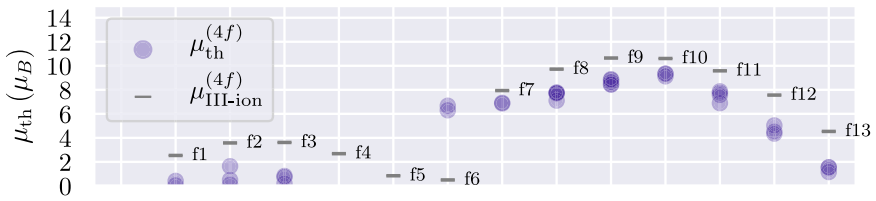

$\mathrm{La} \mathrm{Ce}$ Pr Nd Pm Sm Eu Gd Tb Dy Ho Er Tm Yb

(b.3) Orbital and spin contributions of $\mu_{\mathrm{th}}^{(4 f)}$

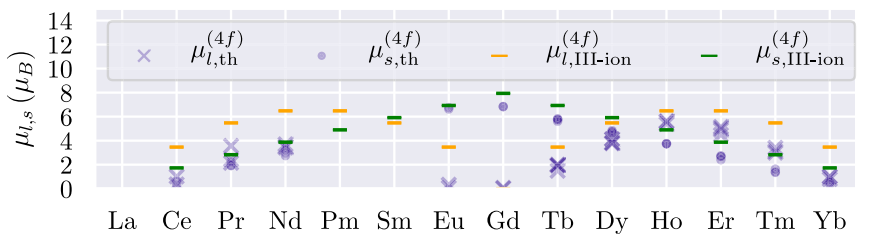

FIG. 5. The magnetic moment per site as a function of electrons per atom for $3 d$ - and $4 f$-orbital magnetism. (a.1),(b.1) The experimental magnetic moment per site $\mu_{\text {expt }}$ (a.2),(b.2) The magnetic moment per site of the CMP + SDFT minimum most similar to experiment with respect to its magnetic configuration $\mu_{\mathrm{th}}$. (a.3),(b.3) Absolute values of the orbital angular momentum contribution $\mu_{l}$ and the spin contribution $\mu_{s}$ to $\mu_{\mathrm{th}}$.

delocalization of metal electrons through covalent bonds with the ligands.

Let us now move on to the case of compounds featuring lanthanides shown in the right-hand column of Fig. 5. As mentioned, in the weak field regime spin-orbit coupling is strong compared to the crystal field effect. Therefore the orbital angular momentum operator $\boldsymbol{L}$ cannot be neglected and the magnetic moment is computed in the $j$ - $j$ coupling scheme in terms of the total angular momentum $\boldsymbol{J}$. In the ionic limit, the electronic ground state can be determined following all three Hund's rules [168] for a given shell configuration $4 f^{n}$ with $n$ electrons. The magnetic moment in terms of the total angular momentum quantum number $j$ then reads

$$
\mu_{j}=g_{j} \sqrt{j(j+1)} \mu_{B},
$$

with the Landé $g$ factor $\left(g_{j}\right)$. Representative, we compute the magnetic moment $\mu_{\mathrm{III}-\text { ion }}^{(4 f)}$ for all $3+$ ions. Note that in fact, $\mathrm{Eu}^{2+}$, for instance, is expected to resemble $\mathrm{Ga}^{3+}$ because both have a $4 f^{7}$ electronic configuration.

Figure 5(b.1) shows the experimental results $\mu_{\text {expt }}^{(4 f)}$ for $4 f$-orbital magnetism in comparison to $\mu_{\mathrm{III}-\text { ion }}^{(4 f)}$. Similar to the $3 d$-orbital magnetism, different compounds featuring the same magnetic element observe vastly different $\mu_{\text {expt }}^{(4 f)}$; however, the origin must be different, as we will see. A comparison to the CMP + SDFT results presented in Fig. 5(b.2) shows good agreement of the overall characteristic behavior. In both experiment and CMP + SDFT, the magnetic moment is just below the ionic limit and a small (large) dome forms in the less (more) than half-filled region.

Noticeably, the compound dependence in the CMP + SDFT results is reduced compared to the experiment. By a more detailed analysis of the experimental data, the compound dependence in $4 f$-orbital magnetism is revealed to arise when long-range order cannot be established very well experimentally. SDFT naturally assumes a wellestablished long-range order by design as it is a zero temperature method. Specific cases are considered in the discussion of Fig. 6.

Figure 5(b.3) shows the absolute value of the spin and orbital contributions $\left(\mu_{s, \mathrm{th}}^{(4 f)}\right.$ and $\left.\mu_{l, \mathrm{th}}^{(4 f)}\right)$ in SDFT. As a reference, we plot a fictitious spin-only $\mu_{s, \text { III-ion }}^{(4 f)}$ and orbitalonly magnetic moment $\mu_{l, \text { III-ion }}^{(4 f)}$ in the ionic limit for $3+$ ions:

$$
\begin{gathered}
\mu_{s, \text { III-ion }}^{(4 f)}=2 \sqrt{s(s+1)} \mu_{B}, \\
\mu_{l, \text { III-ion }}^{(4 f)}=\sqrt{l(l+1)} \mu_{B} .
\end{gathered}
$$

Prominently, the destructive (constructive) coupling for less (more) than half filling is confirmed and visualized. Further, the spin contribution $\mu_{s, \text { th }}^{(4 f)}$ very closely aligns with the ionic limit. This can be expected as $4 f$ electrons barely delocalize by covalently bonding with the surrounding ligands. The orbital contribution $\mu_{l, \text { th }}^{(4 f)}$ shows a clearly 


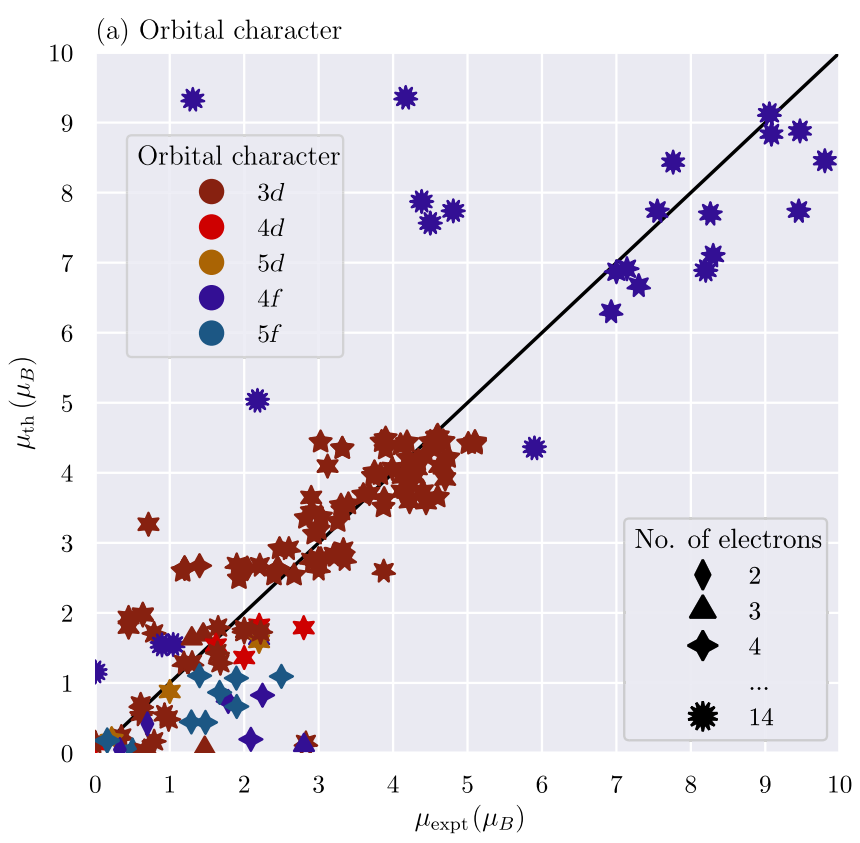

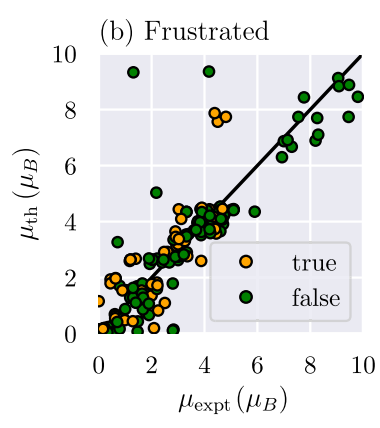
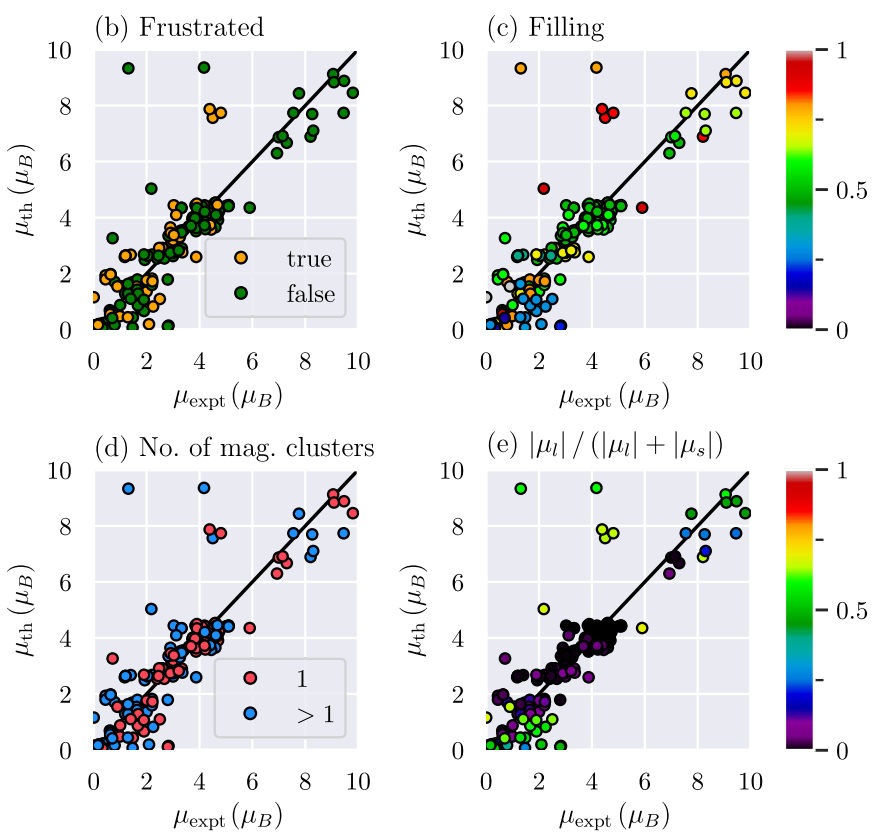

FIG. 6. Average magnetic moment per site of the CMP + SDFT minimum most similar to experiment with respect to its magnetic configuration $\mu_{\mathrm{th}}$ compared to the experimentally measured magnetic moment per site $\mu_{\text {expt }}$. (a) Color indicates the valence orbital character of the magnetic site. The number of points on a star mark equals number of electrons. (b) Color indicates if the material is expected to be frustrated due to odd numbered rings of antiferromagnetic bonds. (c) The color bar shows the filling of the valence orbital of the magnetic site. (d) Color indicates if a single cluster or multiple clusters are magnetic in the material. (e) The color bar shows the normalized orbital angular momentum contribution $\left|\mu_{l}\right| /\left(\left|\mu_{l}\right|+\left|\mu_{s}\right|\right)$.

reduced value compared to $\mu_{l, \text { III-ion }}^{(4 f)}$. This might be interpreted as partial quenching of $\boldsymbol{L}$ in SDFT, which is supported by the observation that the reduction of $\mu_{l, \mathrm{III}-\text { ion }}^{(4 f)}$ is stronger for lighter elements.

So far it has become clear that there is no systematic overestimation of the on-site magnetic moment by $\mathrm{CMP}+\mathrm{SDFT}$. However naively one might anyways expect a general underestimation due to the lack of treatment of strong electronic correlation effects in SDFT, albeit strong electronic correlation is expected in particular in $3 d$ and $4 f$ bands. As we see in the following, the data defy this general expectation of an underestimated on-site moment. To this end, let us compare $\mu_{\text {th }}$ and $\mu_{\text {expt }}$ compoundwise, or rather clusterwise for all compounds.

Figure 6 juxtaposes the average magnetic moment per site $\mu_{\text {th }}$ of the magnetic configuration with MAXOEXP and the experimentally measured magnetic moment per site $\mu_{\text {expt }}$. If for a magnetic cluster $\mu_{\mathrm{th}} \approx \mu_{\text {expt }}$, the data point is in close vicinity to the median and the size of the magnetic moment per site is well estimated. In Fig. 6(a), each cluster $c_{j}$ is represented by a star, whose color indicates the orbital character of the magnetic site and the number of points indicates which magnetic element forms the cluster. For instance, the five-pointed dark red star corresponds to a $\mathrm{Mn}$ cluster, since $\mathrm{Mn}$ atom has five $3 d$ electrons. At first sight, there is no general overestimation or underestimation seen in the scatter plot.
Moreover, the data suggest that the uncertainty of SDFT is reflected in the absolute deviation of $\left|\mu_{\text {th }}-\mu_{\text {expt }}\right|$, rather than some relative deviation of the magnetic moment $\left|\mu_{\text {th }}-\mu_{\text {expt }}\right| /\left|\mu_{\text {th }}+\mu_{\text {expt }}\right|$. Indeed, $51.90 \%$ of the magnetic moments are within $\pm 0.5 \mu_{B}$, and beyond $77.22 \%$ obey $\left|\mu_{\text {th }}-\mu_{\text {expt }}\right| \leq 1 \mu_{B}$. Concomitantly, in the small magnetic moment regime, that is approximately $\mu \lesssim 2 \mu_{B}$, no reliable prediction is possible. In the mid to high magnetic moment regime, on the other hand, a mostly accurate prediction is made.

There is an accumulation of $3 d$ data points within $2 \mu_{B}<\mu_{\mathrm{th}}<5 \mu_{B}$, whose center of mass closely aligns with the median. However, an apparent lack of precision leads to a wide spread around the median. Despite another accumulation of $4 f$ data points in the range of $6 \mu_{B}<\mu_{\text {th }}<$ $10 \mu_{B}$ showing similarly a high accuracy with a center of mass near the median, we also see many outliers with $4 f$-orbital character across the entire range of the on-site magnetic moments.

The specific group of three outliers at $4 \mu_{B}<\mu_{\text {expt }}<5 \mu_{B}$ and $7 \mu_{B}<\mu_{\mathrm{th}}<8 \mu_{B}$ correspond to $\mathrm{Er}$ clusters in $\mathrm{Er}_{2} \mathrm{Sn}_{2} \mathrm{O}_{7}, \mathrm{Er}_{2} \mathrm{Ru}_{2} \mathrm{O}_{7}$, and $\mathrm{Er}_{2} \mathrm{Pt}_{2} \mathrm{O}_{7}$, listed from left to right. In the ionic limit, the ground state electronic configuration of $\mathrm{Er}^{3+}$ is ${ }^{4} I_{15 / 2}$ with the Landé $g$ factor $\left(g_{j}\right)$ of $6 / 5$. Therefore, $\mu_{\mathrm{III}-\text {-ion }}^{(\mathrm{Er})}$ is estimated to be $9.58 \mu_{B}$ using Eq. (24). We see that $\mu_{\text {th }}$ of the three outliers are

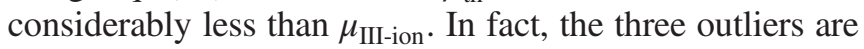


known candidates for realizing a spin-liquid phase due to the presence of magnetic frustration, as described in Refs. [75,122,123] and hence present highly nontrivial cases.

The two outliers with $\mu_{\mathrm{th}}>9 \mu_{B}$ correspond to Ho clusters. Both data points are contributed by the same material $\mathrm{HoMnO}_{3}$, which contains two inequivalent $\mathrm{Ho}$ sites on top of a Mn cluster. The latter orders at $T=78.5 \mathrm{~K}$ and is well estimated by CMP + SDFT with $\mu_{\text {expt }}^{(\mathrm{Mn})}=3.32 \mu_{B}$ and $\mu_{\mathrm{th}}^{(\mathrm{Mn})}=3.47 \mu_{B}$. On the other hand, experimentally ordering of the two Ho clusters is subject to controversy [134,169-172]. It seems unclear from an experimental perspective whether one or both Ho sites order even down to approximately $2 \mathrm{~K}$. Generally, the long-range ordering of magnetic moments on Ho sites is suggested to occur at much lower temperature compared to Mn sites. As mentioned above, a strict comparison of the SDFT result to $\mu_{\text {expt }}$ is inappropriate in the case that proper long-range ordering cannot be established experimentally. Nevertheless, SDFT can be compared to the ionic limit, similar to the discussion on the three materials containing Er. The ground state electronic configuration of $\mathrm{Ho}^{3+}$ is ${ }^{5} I_{8}$, which yields $\mu_{\mathrm{III}-\mathrm{ion}}^{(\mathrm{Ho})}=10.61 \mu_{B}$ as an estimate. To conclude, in $\mathrm{HoMnO}_{3}$ the $\mu_{\mathrm{th}}^{(\mathrm{Ho})}$ of the Ho clusters lie below $\mu_{\mathrm{III}-\mathrm{ion}}^{(\mathrm{Ho})}$ and a strict comparison to $\mu_{\mathrm{expt}}^{(\mathrm{Ho})}$ is inappropriate.

In Fig. 6(b), again $\mu_{\text {th }}$ and $\mu_{\text {expt }}$ are compared, but additionally the color indicates whether or not the compound is expected to be frustrated. Here, the expectation of frustration is based on whether nearest neighbors form rings of odd number of magnetic sites. Assuming AFM coupling this geometrically leads to magnetic frustration. Hence, we take advantage of the database being specifically focused on antiferromagnets. Furthermore, rings of even number of magnetic sites could potentially also yield a magnetically frustrated system, if the AFM coupling is anisotropic, such as in the Kitaev model. We hence note that the definition of expected frustration used here is imprecise and only suitable for a quick superficial classification.

Figure 6(b) shows that indeed the well-estimated $4 f$ clusters in the large magnetic moment regime are not expected to feature magnetic frustration. The discussed group of three outliers, on the other hand, are expected to be frustrated. Data points with $4 f$-orbital character in the small magnetic moment regime $\mu_{\text {th }}<2 \mu_{B}$ are likewise expected to be magnetically frustrated and are not particularly well estimated. Although we expect that $\mu_{\text {th }}$ is overestimated when the system is frustrated, many clusters that are expected to be magnetically frustrated are not necessarily overestimated. And some outliers are-at least in the approximate definition employed here-not expected to be frustrated. However, as we have seen for $\mathrm{HoMnO}_{3}$ there might be other nontrivial phenomena preventing a proper long-range order. Hence, the geometrically expected magnetic frustration is not a sufficient indicator for overestimation of the magnetic moment.

Figure 6(c) displays the filling on a color map from 0 to 1 , where 0.5 corresponds to half filling. Here, the filling is defined as the ratio between the number of $d$ or $f$ electrons in each magnetic atom and the number of orbitals. For the number of electrons, we consider the charge neutral state; i.e., the ionized state is not taken account. Less (more) than half-filled $4 f$ and $5 f$ clusters appear in the underestimated (overestimated) region.

Figure 6(d) addresses the number of magnetic clusters present in a specific compound. The data points corresponding to single cluster (red) and multiple clusters (blue) appear to be evenly distributed. Let us divert the attention toward data points with $\mu_{\mathrm{th}} \approx 0$. It should be noted that these are not paramagnetic solutions. Two scenarios can yield $\mu_{\text {th }} \approx 0$ : Either another cluster bears most of the onsite magnetic moment, or the spin contribution to the magnetic moment $\mu_{s}$ is canceled by the orbital contribution to the magnetic moment $\mu_{l}$.

Figure 6(e) shows the normalized orbital contribution,

$$
\frac{\mu_{l}}{\left|\boldsymbol{\mu}_{l}\right|+\left|\boldsymbol{\mu}_{s}\right|},
$$

in SDFT to the total magnetic moment $\mu_{\mathrm{th}}=\left|\boldsymbol{\mu}_{s}+\boldsymbol{\mu}_{l}\right|$. Below the median in the small magnetic moment regime, indeed many clusters with less than half-filled orbitals observe $\mu_{l} /\left(\left|\boldsymbol{\mu}_{l}\right|+\left|\boldsymbol{\mu}_{s}\right|\right) \approx 0.5$. In these instances, $\boldsymbol{\mu}_{s}$ and $\boldsymbol{\mu}_{l}$ adopt opposing signs and thus the contributions in fact cancel. Clusters of heavier lanthanides are well estimated solely as a result of including $\mu_{l}$. Considering once more Fig. 5(b.3) and a comparison of Figs. 5(a.1) and 5(a.2), the agreement between experiment and SDFT could be improved, if the orbital angular momentum would be less quenched in SDFT.

\section{F. $\mathrm{CMP}+\mathrm{SDFT}+\boldsymbol{U}$ case study}

Hitherto we have discussed the effects of spin-orbit coupling and crystal field splitting on magnetism in compounds with $3 d$ - and $4 f$-orbital character and omitted the careful treatment of another important energy scale in these systems: the electron-electron correlation due to intraorbital Coulomb repulsion $U$. There are various extensions to include electronic correlation beyond SDFT: For instance, SDFT $+U$ [173], SDFT + DMFT [174-179], self-consistent ab initio DГA [180], and other diagrammatic extensions beyond DMFT [181,182]. In fact, these methods have brought important insight in the properties of many compounds closely related to the ones under investigation here [183-186], in particular with respect to Mott-Hubbard localization.

A full treatment of electron-electron correlations from first principles for all materials introduces various 
(a) $U=0 \mathrm{eV}$

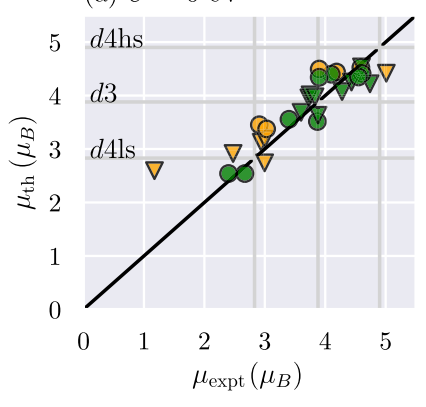

(b) $U=2 \mathrm{eV}$

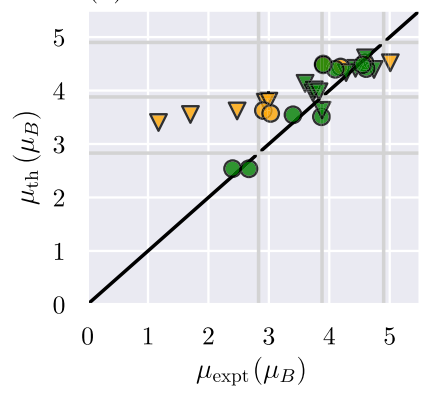

(c) $U=3 \mathrm{eV}$

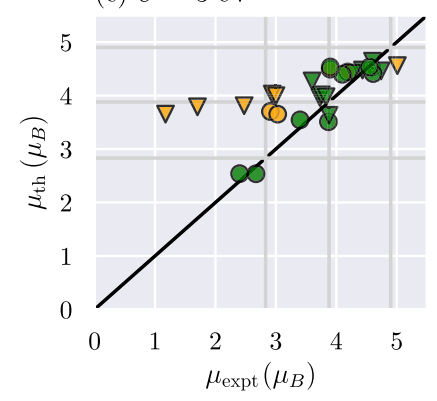

(d) $U=4 \mathrm{eV}$

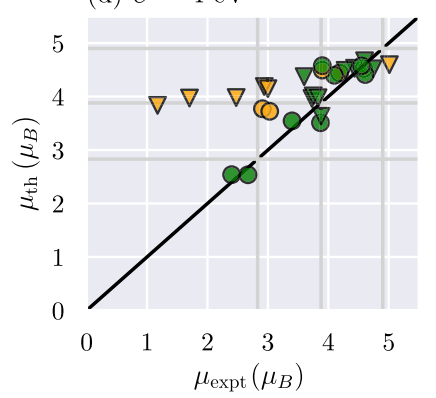

- Frustrated 0 Not frustrated

- Contains oxygen

$\nabla$ No oxygen

FIG. 7. Average magnetic moment per Mn site of the CMP + SDFT $+U$ minimum most similar to the experiment with respect to its configuration $\mu_{\mathrm{th}}$ compared to the experimentally measured magnetic moment per site $\mu_{\text {expt }}$. (a) $U=0 \mathrm{eV}$, (b) $U=2 \mathrm{eV}$, (c) $U=3 \mathrm{eV}$, (d) $U=4 \mathrm{eV}$.

challenges and is beyond the scope of this paper. While it is in principle possible to estimate the parameter $U$ from first principles by means of constraint random phase approximation [187,188], the computational cost of this procedure is immense. Therefore, albeit we aim at the prediction of the magnetic ground state from first principles, we must resort to introducing $U$ as an adjustable parameter in this section. In particular, we will screen $U=2,3,4 \mathrm{eV}$ for $d$ orbitals in $\mathrm{Mn}$ and $U=4,6,8 \mathrm{eV}$ for $f$ orbitals of $\mathrm{Eu}$ and Gd in accordance with the range of typical $U$ values used in literature $[183,186]$. Although this amounts to 1545 additional CMP + SDFT $+U$ calculations, we caution the reader that our efforts to include $U$ may not be conclusive enough to be generalized to statements about the importance of strong electronic correlations in regard to the prediction of the magnetic ground state.

We have chosen to perform CMP $+\mathrm{SDFT}+U$ calculations for all materials containing a single Mn cluster because of the following reasons. (i) It is a well-defined subgroup of 28 materials, which is near the minimum sample size necessary to obtain statistically significant results. (ii) The compounds are not prone to a spin-liquid ground state, so that the comparison with the experiment stands on solid grounds. (iii) For $U=0$ the size of the magnetic moment is overestimated or underestimated depending on the material, as shown in Fig. 7(a). Therefore, we can clearly distinguish if the theoretical magnetic moment $\mu_{\text {th }}$ gets closer to the experimental value $\mu_{\text {expt }}$ with increasing $U$ or if $\mu_{\text {th }}$ increases regardless of whether it was already overestimated for $U=0$. (iv) The total energy distribution $\rho_{\mathrm{Mn}, \mathrm{MAXOEXP}}$ of the CMP + SDFT minimum that yields the maximum overlap with the experiment (MAXOEXP) for $U=0$ has a strong bias toward the energy minimum. In other words, there is room to improve if MAXOEXP were to always agree with the CMP + SDFT global minimum and also room to deteriorate if $\rho_{\mathrm{Mn}, \mathrm{MAXOEXP}}$ were to spread across a wider range of energy.
Furthermore, we have chosen to perform $\mathrm{CMP}+$ $\mathrm{SDFT}+U$ calculations for all materials containing $\mathrm{Eu}$ and Gd, which are the following four compounds: $\mathrm{EuTiO}_{3}$, $\mathrm{EuZrO}_{3}, \mathrm{GdVO}_{4}, \mathrm{GdB}_{4}$. That is because these $4 f$ elements are close to half filling, where the orbital contribution to the on-site magnetic moment $\mu_{l}$ vanishes, as can be confirmed in Fig. 5(b.3). Thus, spin-orbit coupling is of no importance in these systems and, furthermore, the crystal field splitting is expected to be small because the strongly localized $4 f$ orbitals are well shielded by the outer $3 d$ and $4 s$ orbitals. Hence, we expect the Coulomb interaction $U$ to predominantly determine the dynamics of $f$ electrons in these compounds.

The two main questions are as follows: (i) Does including $U$ improve the prediction of the most stable magnetic structure and (ii) will the estimation of the on-site magnetic moment improve upon introducing $U$ ? Without further ado let us present the results of CMP $+\mathrm{SDFT}+U$ for compounds containing $\mathrm{Mn}, \mathrm{Eu}$, and $\mathrm{Gd}$.

We find that CMP + SDFT $+U$ identifies the same local minima as CMP + SDFT with different relative total energy to each other. Thus, MAXOEXP is the same at any value of $U$. Moreover, the range of the total energy distribution $\rho_{\mathrm{Mn} \text {,tot }}$ is $U$ independent and ranges from 0 to $1000 \mathrm{meV}$. Hence, we ask if MAXOEXP tends to have the lowest total energy and if this tendency is increased by increasing $U$. We note that for the limited number of materials investigated, the total energy distribution of MAXOEXP $\rho_{\text {Mn,MAXOEXP }}$ for $U=0,2 \mathrm{eV}$ ranges from $0 \mathrm{meV}$ to approximately $5 \mathrm{meV}$. Additionally the distribution of $\rho_{\text {Mn,MAXOEXP }}$ is skewed toward lower total energy compared to $\rho_{\mathrm{Mn}, \text { tot }}$. On the other hand, for $U=3,4 \mathrm{eV}$ $\rho_{\text {Mn,MAXOEXP }}$ reaches close to $1000 \mathrm{meV}$, while it remains skewed toward lower total energy. In other words, introducing $U$ does not assign the correct total energy to the true magnetic ground state found in the experiment in this data. In fact, increasing $U$ reduces the tendency for MAXOEXP to have a particularly low total energy. 
Let us now discuss the estimation of the on-site magnetic moment. Figures 7(a)-7(d) show the average on-site magnetic moment $\mu_{\mathrm{th}}$ of MAXOEXP for all materials containing a single Mn cluster for $U=0,2,3$, and 4, respectively. The gray lines labeled $d 4$ hs (high spin), $d 3$, and $d 4$ ls (low spin) correspond to the spin-only contribution of Mn with formal oxidation $3+$ and $4+$, i.e., 4 and $3 d$ electrons, in an octahedral ligand field [165], same as in Fig. 5(a.3). We see that increasing $U$ never decreases $\mu_{\text {th }}$. For each compound we distinguish whether the crystal contains loops of odd number of magnetic sites and is thus expected to be frustrated. This is indicated by the color of the marker. The shape of the marker implies if the compound contains oxygen. Note that GGA is known to cause overbinding of oxygen to transition metals [189-192]. The effect of increasing $U$ most strongly increases $\mu_{\text {th }}$ of frustrated compounds containing no oxygen that are far away from the high-spin state for $U=0 \mathrm{eV}$. The increase of $\mu_{\text {th }}$ also seems to occur-though less pronounced-in compounds that satisfy only one of the conditions. That is either compounds that are expected to be frustrated albeit containing oxygen or compounds lacking oxygen, although they are not expected to be frustrated.

We speculate that the overbinding of the ligand oxygen could lead to a very strong crystal field splitting. This may protect the low-spin state, for instance, of the compounds near $\mu_{\text {th }} \approx 2.5 \mu_{B}$. Furthermore, we intuitively expect frustration to reduce the size of the magnetic moment, because not all AFM bonds can be satisfied simultaneously and the cost of not satisfying a bond is proportional to the size of the on-site magnetic moment. Introducing $U$ has a localizing effect and might cause intra-atomic effects to become prevalent over frustration. The on-site magnetic moment is reduced compared to the ionic limit due to delocalization of the Mn electrons, for instance, onto the ligands. Moreover, itinerancy may lead to a reduced $\mu_{\text {th }}$ compared to the ionic limit depending on the partial density of states. Thus, there are various reasons for the on-site magnetic moment ranging from $1 \mu_{B}$ to $5 \mu_{B}$. In the investigated Mn compounds the agreement of $\mu_{\text {th }}$ with $\mu_{\text {expt }}$ corroded by introducing $U$ by means of GGA $+U$.

The CMP $+\mathrm{SDFT}+U$ results for $\mathrm{EuTiO}_{3}, \mathrm{EuZrO}_{3}$, $\mathrm{GdVO}_{4}$, and $\mathrm{GdB}_{4}$ similarly show no improvement by introducing $U$. In fact, for $\mathrm{EuTiO}_{3}, \mathrm{EuZrO}_{3}$, and $\mathrm{GdVO}_{4}$ the magnetic ground state is falsely predicted to be ferromagnetic for $U>4 \mathrm{eV}$. Again the same local minima are found, so that in these cases MAXOEXP observes increasing total energy by increasing $U$ relative to the $\mathrm{CMP}+\mathrm{SDFT}+U$ global minimum at each $U$ value. For $\mathrm{GdB}_{4}$ the CMP + SDFT $+U$ global minimum is AFM along the $c$ direction for all $U$ values, while the experimental structure is a hexadecapole in the $a b$ plane. However, for $U=0 \mathrm{eV}$ these two magnetic structures are almost degenerate with $0.5 \mathrm{meV}$ difference between their total energies. Furthermore, for increasing $U$ the system increasingly prefers the out-of-plane magnetic structure.

The on-site magnetic moment is increased with increasing $U$ for all four compounds containing Eu and $\mathrm{Gd}$. As can be seen in Fig. 6(a) around $\mu_{\text {expt }} \approx 7 \mu_{B}$, the size of $\mu_{\text {th }}$ is slightly underestimated for $U=0$ for all four compounds. Thus, the estimates of $\mu_{\mathrm{th}}=6.95 \mu_{B}, 6.98 \mu_{B}, 7.04 \mu_{B}$, and $7.10 \mu_{B}$ for $U=8 \mathrm{eV}$ for $\mathrm{EuTiO}_{3}, \mathrm{EuZrO}_{3}, \mathrm{GdVO}_{4}$, and $\mathrm{GdB}_{4}$, respectively, are closer to the experimental values $\mu_{\text {expt }}=6.93 \mu_{B}, 7.30 \mu_{B}, 7.00 \mu_{B}$, and $7.14 \mu_{B}$ than for $U=0 \mathrm{eV}, \mu_{\mathrm{th}}=6.30 \mu_{B}, 6.67 \mu_{B}, 6.87 \mu_{B}$, and $6.91 \mu_{B}$. Let us note that other $4 f$ compounds slightly overestimated onsite magnetic moment and we suspect for these compounds increasing $U$ would also increase $\mu_{\text {th }}$.

Instead of focusing on effects of strong electronic correlations, we speculate that the prediction of the true experimental magnetic ground state could be improved by a different choice of exchange-correlation functional. We would like to point out one recent example of a detailed SDFT $+U$ study [193] on $\mathrm{LiOsO}_{3}$ and $\mathrm{NaOsO}_{3}$ testing other exchange-correlation functionals thus far implemented in VASP, including local spin-density approximation, PBE's improved version for solids (PBEsol), the strongly constrained appropriately normed (SCAN) meta-GGA functional, and hybrid functional HSE06. By means of scanning different $U$ values including predicted ones from constraint random phase approximation, Liu et al. found that none of the considered functionals is capable to simultaneously predict the correct magnetic ground state for $\mathrm{LiOsO}_{3}$ and $\mathrm{NaOsO}_{3}$ comparing the total energy of two energetically favorable configurations. The treatment of exchange-correlation effects in all of these functionals hitherto implemented in VASP has the underlying assumption that locally the spin density can be diagonalized. Schematically, an electron thus only couples to an exchange-correlation magnetic field that is parallel to its own magnetization. In the past two decades, some work [194-206] has been done to extend SDFT to include the socalled spin-torque effect, which couples the electron's spin to the exchange-correlation magnetic field including antisymmetric terms.

\section{CONCLUSION AND OUTLOOK}

This study is a benchmark of an ab initio prediction of the magnetic ground state using a novel approach termed CMP + SDFT. This scheme devises a combination of the cluster-multipole expansion and the spin-density functional theory for noncollinear magnetism. We find that materials existent in nature are well described in terms of only few CMPs and infer the CMP basis to be a suitable basis for magnetic configurations. Additionally, the experimental data suggest that the magnetic ground state favors either pure CMPs or linear combinations of CMPs having the same expansion order and same irreducible representation. Guided by this heuristic rule an exhaustive list of initial 
candidate magnetic configurations for $a b$ initio calculations in the framework of SDFT is created.

A high-throughput calculation of $2935 a b$ initio calculations using VASP led to a handful of CMP + SDFT local minima corresponding to different possible magnetic configurations for each material. $90.16 \%$ of materials yield the experimental magnetic space group for at least one of the $\mathrm{CMP}+$ SDFT local minima. Furthermore, the maximum overlap between the experimental magnetic configuration and the CMP + SDFT local minima exceeds 0.75-with 1 corresponding to equivalence-in $70.99 \%$ of all materials.

An $a b$ initio prediction of the most stable magnetic configuration in the experiment is guided by a comparison of the total energy in SDFT using GGA of the possible magnetic configurations for each material. In particular, the local minimum with the largest overlap with the experiment (MAXOEXP) is expected to yield the lowest total energy. Indeed, for materials featuring magnetic sites with $d$-orbital magnetism, MAXOEXP is in great majority of the cases less than $1 \mathrm{meV}$ above the so-called CMP + SDFT global minimum. On the other hand, the same could not be confirmed for $f$-orbital magnetism. In fact, MAXOEXP for $f$-orbital magnetism shows no tendency toward lower total energy. The implementation of GGA PBE [207] used in this study did not necessarily assign the lowest total energy to the local minimum with the largest overlap with the experiment.

We have further investigated the effect of including strong electronic correlations on the level of SDFT $+U$ for materials containing a single Mn cluster, Eu cluster, or Gd cluster. Our results show that for the materials we investigated introducing $U$ has a rather unfavorable influence on the prediction for both the magnetic ground state and the size of the magnetic moment. In the end of Sec. III E, we speculate that the prediction of the true experimental magnetic ground state could be improved by a different choice of exchange-correlation functional that accounts for the spin-torque effect [194-206], as opposed to focusing on effects of strong electronic correlations.

As far as we know, the only other scheme that aims at the prediction of noncollinear magnetic structures is based on a genetic algorithm by Zheng and Zhang [14]. In their approach only the fittest magnetic structures of each generation survive, which is decided based on the total energy of the magnetic structure. Thus, currently it converges to the global minimum corresponding to a theoretical magnetic ground state that is not necessarily the true magnetic ground state found in the experiment. On the other hand, in CMP + SDFT we yield a set of magnetic configurations that are local minima of the total energy, which is very likely to include the magnetic ground state as we have demonstrated in this paper. Hence, we want to emphasize that CMP + SDFT succeeded to significantly narrow down the number of possible magnetic ground states. This is achieved thanks to a list of candidate magnetic configurations that is tailored to account for details of the symmetry of the crystallographic unit cell. In fact, CMP theory enables SDFT to identify local minima from a feasible number of candidate magnetic configurations that put data screening and AFM material design within reach. On average, in this study we performed only $2935 / 131=22.4$ for each material, while in Ref. [14] they performed 30 calculations in each generation. In order to ensure convergence, they ran the evolution for 30 generations, which amounts to 900 calculations for one material. This comparison of the number of calculations that are necessary to find the theoretical magnetic ground state emphasizes that our list of candidates - the CMP basis combined with our heuristic rule and omitting the magnetic configurations corresponding to different magnetic domains of the same magnetic structure-is well suited to search the space of all possible magnetic configurations.

In addition, this study showed that the on-site magnetic moment could be estimated surprisingly well by GGA without including $U$. The precision of the predicted magnetic moment is estimated to be roughly $\pm 0.5 \mu_{B}$. Some outliers arise from a lack of long-range order in the experiment. This can be due to extremely low transition temperatures and magnetic frustration. Despite some explainable outliers, the prediction shows no major systematic overestimation or underestimation of the on-site magnetic moment in GGA. In contrast to the experiment, the SDFT calculation grants additional insight into the balance of spin contribution and orbital angular momentum contribution to the total magnetic moment. The first row transition metals prove to be well described by RusselSaunders coupling applicable within the strong field regime. In other words, the orbital angular momentum is quenched and the spin-only ionic limit can be used as a reference. The case of lanthanides, on the other hand, is representative for systems in the weak field regime. The onsite magnetic moment is well described in the $j$ - $j$ coupling scheme. In the end of Sec. III D, we speculate that GGA might have slightly overestimated the crystal field effects compared to the strength of spin-orbit coupling. Some related discussions of GGA causing an overbinding of ligand oxygen can be found in the literature [189-192]. This could explain why materials governed by crystal field splitting - such as the compounds with $d$-orbital magnetism - are assigned more appropriate total energy by GGA. Yet, in materials governed by spin-orbit couplingsuch as lanthanides - the experimental magnetic configuration is not assigned the lowest total energy by GGA. The balance between spin-orbit coupling and crystal field splitting becomes particularly crucial for lighter $4 f$ elements and heavier $3 d$ elements, where the orbital angular momentum is only partially quenched.

We conclude by putting this study into a bigger context and providing an outlook into future works. The starting 
point of this study was the experimental database MAGNDATA [39]. It conveniently facilitated testing and benchmarking of our $a b$ initio scheme to predict the magnetic ground state. Generally, experimental databases [208-217] not only facilitate testing and benchmarking of theoretical methods, but also data mining in the experimentally explored chemical space. Indeed, for some nonmagnetic functional materials an informed search and optimization has led to promising discoveries [218-230]. However, so far, apart from a few pioneering works [9-13] that are constrained to specific cases, these breakthroughs in material design have not yet been matched by similar advances with respect to AFM materials. Certainly one of the major obstacles is that compared to databases of crystal structures with more than 200000 entries, MAGNDATA has to date a modest amount of about 1130 entries. This is because the experimental determination of the magnetic configuration is much more involved than that of the crystal structure. Given this situation, it is an urgent challenge to construct a large-scale computational database of AFM materials. The presented benchmark provides a crucial step in laying a solid foundation for the construction of such a computational database of AFM materials. We are optimistic that $a b$ initio calculations will soon be able to reliably predict the magnetic ground state. Based on that, our CMP + SDFT scheme will be able to construct a computational database of magnetic materials with a feasible amount of computational effort. On top of that database, model calculations-using, for instance, the Liechtenstein method [231-233]—can lead to useful insights in particular with respect to the spin-wave dispersion and critical temperatures of magnetic phase transitions. Finally, let us note that many magnetic transitions are accompanied by structural transitions. And it might prove imperative to follow a scheme of successively relaxing the atomic position and the magnetic ground state. In the current study we avoided this obstacle by using the atomic positions obtained experimentally. However, in view of material design, the ability to treat experimentally unknown crystal structures will be of great use.

\section{ACKNOWLEDGMENTS}

We are thankful for useful discussions with Stephan Huebsch, Takashi Koretsune, and Saeed Bahramy. Moreover, we gratefully acknowledge the Center for Computational Materials Science, Institute for Materials Research, Tohoku University for the use of MASAMUNE-IMR (MAterials science Supercomputing system for Advanced MUlti-scale simulations towards NExt-generation-Institute for Materials Research) (Project No. 19S0005). This work was supported by a Grant-in-Aid for Scientific Research (No. 19H05825 and No. 16H06345) from the Ministry of Education, Culture, Sports, Science and Technology, and CREST (JPMJCR18T3) from the Japan Science and Technology
Agency, and JSPS KAKENHI Grants No. JP15H05883 (J-Physics), No. JP19H01842, No. JP20H05262, and No. JP20K21067, and JST PRESTO Grants No. JPMJPR17N8 and No. JPMJPR20L7.

[1] G. R. Schleder, A. C. Padilha, C. M. Acosta, M. Costa, and A. Fazzio, From DFT to Machine Learning: Recent Approaches to Materials Science-A Review, J. Phys. Mater. 2, 032001 (2019).

[2] H. Zhang, High-Throughput Design of Magnetic Materials, Electron. Struct. (2020) https://doi.org/10.1088/25161075/abbb25.

[3] V. Baltz, A. Manchon, M. Tsoi, T. Moriyama, T. Ono, and Y. Tserkovnyak, Antiferromagnetic Spintronics, Rev. Mod. Phys. 90, 015005 (2018).

[4] T. Nomoto and R. Arita, Cluster Multipole Dynamics in Noncollinear Antiferromagnets, Phys. Rev. Research 2, 012045(R) (2020).

[5] W. Kleiner, Space-Time Symmetry of Transport Coefficients, Phys. Rev. 142, 318 (1966).

[6] W. Kleiner, Space-Time Symmetry Restrictions on Transport Coefficients. II. Two Theories Compared, Phys. Rev. 153, 726 (1967).

[7] M. Seemann, D. Ködderitzsch, S. Wimmer, and H. Ebert, Symmetry-Imposed Shape of Linear Response Tensors, Phys. Rev. B 92, 155138 (2015).

[8] J. Kübler, Theory of Itinerant Electron Magnetism (Oxford University Press, New York, 2017), Vol. 106.

[9] M. K. Horton, J. H. Montoya, M. Liu, and K. A. Persson, High-Throughput Prediction of the Ground-State Collinear Magnetic Order of Inorganic Materials Using Density Functional Theory, npj Comput. Mater. 5, 64 (2019).

[10] S. Sanvito, C. Oses, J. Xue, A. Tiwari, M. Zic, T. Archer, P. Tozman, M. Venkatesan, M. Coey, and S. Curtarolo, Accelerated Discovery of New Magnets in the Heusler Alloy Family, Sci. Adv. 3, e1602241 (2017).

[11] V. Stevanović, S. Lany, X. Zhang, and A. Zunger, Correcting Density Functional Theory for Accurate Predictions of Compound Enthalpies of Formation: Fitted Elemental-Phase Reference Energies, Phys. Rev. B 85, 115104 (2012).

[12] P. Gorai, E. S. Toberer, and V. Stevanović, Thermoelectricity in Transition Metal Compounds: The Role of Spin Disorder, Phys. Chem. Chem. Phys. 18, 31777 (2016).

[13] Y. Xu, L. Elcoro, Z. Song, B. J. Wieder, M. G. Vergniory, N. Regnault, Y. Chen, C. Felser, and B. A. Bernevig, HighThroughput Calculations of Antiferromagnetic Topological Materials from Magnetic Topological Quantum Chemistry, Nature (London) 586, 702 (2020).

[14] F. Zheng and P. Zhang, MagGene: A Genetic Evolution Program for Magnetic Structure Prediction, arXiv:2003 .05650 .

[15] M.-T. Suzuki, T. Koretsune, M. Ochi, and R. Arita, Cluster Multipole Theory for Anomalous Hall Effect in Antiferromagnets, Phys. Rev. B 95, 094406 (2017).

[16] M.-T. Suzuki, H. Ikeda, and P. M. Oppeneer, FirstPrinciples Theory of Magnetic Multipoles in Condensed Matter Systems, J. Phys. Soc. Jpn. 87, 041008 (2018). 
[17] V.T. N. Huyen, M.-T. Suzuki, K. Yamauchi, and T. Oguchi, Topology Analysis for Anomalous Hall Effect in the Noncollinear Antiferromagnetic States of $\mathrm{Mn}_{3} A \mathrm{~N}$ $(A=\mathrm{Ni}, C u, Z n, G a, G e, P d, I n, S n, I r, P t)$, Phys. Rev. B 100, 094426 (2019).

[18] M. T. Suzuki, T. Nomoto, R. Arita, Y. Yanagi, S. Hayami, and H. Kusunose, Multipole Expansion for Magnetic Structures: A Generation Scheme for a Symmetry-Adapted Orthonormal Basis Set in the Crystallographic Point Group, Phys. Rev. B 99, 174407 (2019).

[19] U. von Barth and L. Hedin, A Local Exchange-Correlation Potential for the Spin Polarized Case, J. Phys. C 5, 1629 (1972).

[20] D. Hobbs and J. Hafner, Ab Initio Density Functional Study of Phase Stability and Noncollinear Magnetism in Mn, J. Phys. Condens. Matter 13, L681 (2001).

[21] A. Dal Corso and A. M. Conte, Spin-Orbit Coupling with Ultrasoft Pseudopotentials: Application to $\mathrm{Au}$ and Pt, Phys. Rev. B 71, 115106 (2005).

[22] TheELK FP-LAPW code, 2020, http://elk.sourceforge.net/.

[23] F. G. Eich and E. K. U. Gross, Transverse Spin-Gradient Functional for Noncollinear Spin-Density-Functional Theory, Phys. Rev. Lett. 111, 156401 (2013).

[24] J. K. Dewhurst, S. Sharma, and C. Ambrosch-Draxl, EXITING code, 2020, http://exciting.sourceforge.net.

[25] S. Sharma, J. K. Dewhurst, C. Ambrosch-Draxl, S. Kurth, N. Helbig, S. Pittalis, S. Shallcross, L. Nordström, and E. K. U. Gross, First-Principles Approach to Noncollinear Magnetism: Towards Spin Dynamics, Phys. Rev. Lett. 98, 196405 (2007).

[26] L. Nordström and D. J. Singh, Noncollinear Intra-atomic Magnetism, Phys. Rev. Lett. 76, 4420 (1996).

[27] H. Eschrig and V.D.P. Servedio, Relativistic Density Functional Approach to Open Shells, J. Comput. Chem. 20, 23 (1999).

[28] G. Kresse and J. Hafner, Ab Initio Molecular Dynamics for Liquid Metals, Phys. Rev. B 47, 558 (1993).

[29] G. Kresse and J. Hafner, Ab Initio Molecular-Dynamics Simulation of the Liquid-Metalamorphous-Semiconductor Transition in Germanium, Phys. Rev. B 49, 14251 (1994).

[30] H. Kusunose, Description of Multipole in $f$-Electron Systems, J. Phys. Soc. Jpn. 77, 064710 (2008).

[31] S. Hayami, M. Yatsushiro, Y. Yanagi, and H. Kusunose, Classification of Atomic-Scale Multipoles under Crystallographic Point Groups and Application to Linear Response Tensors, Phys. Rev. B 98, 165110 (2018).

[32] T. Hahn and H. Klapper, Crystallographic and Noncrystallographic Point Groups, in International Tables for Crystallography (2006), Vol. A, pp. 762-803, https:// doi.org/10.1107/97809553602060000520.

[33] In other words, $\mathbf{e}_{i}^{(n)}$ correspond to components of $\left\{\boldsymbol{e}_{l \gamma}^{\mu}\right\}$ in Ref. [18] except that here $\sum_{i}^{N} \mathbf{e}_{i}^{(n)} \mathbf{e}_{i}^{\left(n^{\prime}\right)}=N \delta_{n n^{\prime}}$, while in Ref. [18] $\left(\left\{\boldsymbol{e}^{n}\right\} \cdot\left\{\boldsymbol{e}^{n^{\prime}}\right\}\right)=\delta_{n n^{\prime}}$. Also note that $\left\{\boldsymbol{e}_{l \gamma}^{\mu}\right\}$ is labeled by $\mu=1,2$ representing magnetic (M) and magnetic toroidal (MT) multipoles, respectively. As we do not expand in terms of MT multipoles, those components appear as higher order magnetic multipoles here, and thus completeness is still ensured.

[34] See Supplemental Material at http://link.aps.org/ supplemental/10.1103/PhysRevX.11.011031 for details of the VASP setup, a complete list of the MAGNDATA entries used in this paper, and the definition of the reproducibility.

[35] J. P. Perdew, K. Burke, and M. Ernzerhof, Generalized Gradient Approximation Made Simple, Phys. Rev. Lett. 77, 3865 (1996).

[36] S. P. Ong, W. D. Richards, A. Jain, G. Hautier, M. Kocher, S. Cholia, D. Gunter, V. L. Chevrier, K. A. Persson, and G. Ceder, Python Materials Genomics (pymatgen): A Robust, Open-Source Python Library for Materials Analysis, Comput. Mater. Sci. 68, 314 (2013).

[37] A. Togo and I. Tanaka, Spglib: A Software Library for Crystal Symmetry Search, arXiv:1808.01590.

[38] A. Yanase, Fortran Program for Space Group (1985).

[39] S. V. Gallego, J. M. Perez-Mato, L. Elcoro, E. S. Tasci, R. M. Hanson, M. I. Aroyo, and G. Madariaga, MAGNDATA: Towards a Database of Magnetic Structures, J. Appl. Crystallogr. 49, 1941 (2016).

[40] F. Moussa, M. Hennion, J. Rodriguez-Carvajal, H. Moudden, L. Pinsard, and A. Revcolevschi, Spin Waves in the Antiferromagnet Perovskite $\mathrm{LaMnO}_{3}$ : A Neutron-Scattering Study, Phys. Rev. B 54, 15149 (1996).

[41] Y. Tokunaga, S. Iguchi, T. Arima, and Y. Tokura, Magnetic-Field-Induced Ferroelectric State in $\mathrm{DyFeO}_{3}$, Phys. Rev. Lett. 101, 097205 (2008).

[42] J. S. White, T. Honda, K. Kimura, T. Kimura, C. Niedermayer, O. Zaharko, A. Poole, B. Roessli, and M. Kenzelmann, Coupling of Magnetic and Ferroelectric Hysteresis by a Multicomponent Magnetic Structure in $\mathrm{Mn}_{2} \mathrm{GeO}_{4}$, Phys. Rev. Lett. 108, 077204 (2012).

[43] M. Reehuis, C. Ulrich, K. Prokeš, S. Mat'aš, J. Fujioka, S. Miyasaka, Y. Tokura, and B. Keimer, Structural and Magnetic Phase Transitions of the Prthovanadates $\mathrm{RVO}_{3}(R=\mathrm{Dy}, \mathrm{Ho}, \mathrm{Er})$ as Seen via Neutron Diffraction, Phys. Rev. B 83, 064404 (2011).

[44] E. Morosan, J. A. Fleitman, Q. Huang, J. W. Lynn, Y. Chen, X. Ke, M. L. Dahlberg, P. Schiffer, C. R. Craley, and R. J. Cava, Structure and Magnetic Properties of the $\mathrm{Ho}_{2} \mathrm{Ge}_{2} \mathrm{O}_{7}$ Pyrogermanate, Phys. Rev. B 77, 224423 (2008).

[45] E. Krén, G. Kádár, L. Pál, and P. Szabó, Investigation of the First-Order Magnetic Transformation in $\mathrm{Mn}_{3} \mathrm{Pt}, \mathrm{J}$. Appl. Phys. 38, 1265 (1967).

[46] M. Fiebig, D. Fröhlich, and H. J. Thiele, Determination of Spin Direction in the Spin-Flop Phase of $\mathrm{Cr}_{2} \mathrm{O}_{3}$, Phys. Rev. B 54, R12681 (1996).

[47] E. Bertaut, L. Corliss, F. Forrat, R. Aleonard, and R. Pauthenet, Etude de Niobates et Tantalates de Metaux de Transition Bivalents, J. Phys. Chem. Solids 21, 234 (1961).

[48] M. Pernet, D. Elmale, and J.-C. Joubert, Structure Magnetique du Metaborate de fer $\mathrm{FeBO}_{3}$, Solid State Commun. 8, 1583 (1970).

[49] R. Plumier, M. Sougi, and R. Saint-James, NeutronDiffraction Reinvestigation of $\mathrm{NiCO}_{3}$, Phys. Rev. B 28, 4016 (1983).

[50] P. J. Brown, P. J. Welfordt, and J. B. Forsyth, Magnetization Density and the Magnetic Structure of Cobalt Carbonate, J. Phys. C 6, 1405 (1973).

[51] P. J. Brown and J. B. Forsyth, The Spatial Distribution of Ferromagnetic Moment in $\mathrm{MnCO}_{3}$, Proc. Phys. Soc. 92, 125 (1967). 
[52] R. A. Alikhanov, Neutron Diffraction Investigation of the Antiferromagnetism of the Carbonates of Manganese and Iron, Sov. Phys. JETP 9, 1204 (1959).

[53] S. M. Disseler, J. A. Borchers, C. M. Brooks, J. A. Mundy, J. A. Moyer, D. A. Hillsberry, E. L. Thies, D. A. Tenne, J. Heron, M. E. Holtz, J. D. Clarkson, G. M. Stiehl, P. Schiffer, D. A. Muller, D. G. Schlom, and W. D. Ratcliff, Magnetic Structure and Ordering of Multiferroic Hexagonal $\mathrm{LuFeO}_{3}$, Phys. Rev. Lett. 114, 217602 (2015).

[54] O. Mentré, M. Kauffmann, G. Ehora, S. Daviero-Minaud, F. Abraham, and P. Roussel, Structure, Dimensionality and Magnetism of New Cobalt Oxyhalides, Solid State Sci. 10, 471 (2008).

[55] B. C. Melot, B. Paden, R. Seshadri, E. Suard, G. Nénert, A. Dixit, and G. Lawes, Magnetic Structure and Susceptibility of $\mathrm{CoSe}_{2} \mathrm{O}_{5}$ : An Antiferromagnetic Chain Compound, Phys. Rev. B 82, 014411 (2010).

[56] R. Troć, M. Pasturel, O. Tougait, A. P. Sazonov, A. Gukasov, C. Sułkowski, and H. Noël, Single-Crystal Study of the Kagome Antiferromagnet $\mathrm{U}_{3} \mathrm{Ru}_{4} \mathrm{Al}_{12}$, Phys. Rev. B 85, 064412 (2012).

[57] M. Reynaud, G. Rousse, J.-N. Chotard, J. RodríguezCarvajal, and J.-M. Tarascon, Marinite $\mathrm{Li}_{2} M\left(\mathrm{SO}_{4}\right)_{2}$ $(M=\mathrm{Co}, \mathrm{Fe}, \mathrm{Mn})$ and $\mathrm{Li}_{1} \mathrm{Fe}\left(\mathrm{SO}_{4}\right)_{2}$ : Model Compounds for Super-Super-Exchange Magnetic Interactions, Inorg. Chem. 52, 10456 (2013).

[58] K. Tsuzuki, Y. Ishikawa, N. Watanabe, and S. Akimoto, Neutron Diffraction and Paramagnetic Scattering from a High Pressure Phase of $\mathrm{MnGeO}_{3}$ (Ilmenite), J. Phys. Soc. Jpn. 37, 1242 (1974).

[59] A. T. Aldred, B. D. Dunlap, D. J. Lam, G. H. Lander, M. H. Mueller, and I. Nowik, Magnetic Properties of Neptunium Laves Phases: $\mathrm{NpMn}_{2}, \mathrm{NpFe}_{2}, \mathrm{NpCo}_{2}$, and $\mathrm{NpNi}_{2}$, Phys. Rev. B 11, 530 (1975).

[60] B. C. Melot, G. Rousse, J.-N. Chotard, M. Ati, J. Rodríguez-Carvajal, M. C. Kemei, and J.-M. Tarascon, Magnetic Structure and Properties of the Li-Ion Battery Materials $\mathrm{FeSO}_{4} \mathrm{~F}$ and $\mathrm{LiFeSO}_{4} \mathrm{~F}$, Chem. Mater. 23, 2922 (2011).

[61] Y. J. Choi, H. T. Yi, S. Lee, Q. Huang, V. Kiryukhin, and S.-W. Cheong, Ferroelectricity in an Ising Chain Magnet, Phys. Rev. Lett. 100, 047601 (2008).

[62] M. Hase, H. Kuroe, V. Y. Pomjakushin, L. Keller, R. Tamura, N. Terada, Y. Matsushita, A. Dönni, and T. Sekine, Magnetic Structure of the Spin- $\frac{1}{2}$ Frustrated QuasiOne-Dimensional Antiferromagnet $\mathrm{Cu}_{3} \mathrm{Mo}_{2} \mathrm{O}_{9}$ : Appearance of a Partially Disordered State, Phys. Rev. B 92, 054425 (2015).

[63] A. Lappas, A. S. Wills, M. A. Green, K. Prassides, and M. Kurmoo, Magnetic Ordering in the Rutile Molecular Magnets, Phys. Rev. B 67, 144406 (2003).

[64] Z.-G. Ye, P. Schobinger-Papamantellos, S.-Y. Mao, C. Ritter, E. Suard, M. Sato, and H. Schmid, A Neutron Diffraction Study of the Magnetic Structure and Phase Transition in $\mathrm{Ni}_{3}^{11} \mathrm{~B}_{7} \mathrm{O}_{1} 3 \mathrm{Cl}$ Boracite, Ferroelectrics 204, 83 (1997).

[65] G. Gitgeatpong, Y. Zhao, M. Avdeev, R. O. Piltz, T. J. Sato, and K. Matan, Magnetic Structure and Dzyaloshinskii-Moriya Interaction in the $S=\frac{1}{2}$
Helical-Honeycomb Antiferromagnet $\alpha-\mathrm{Cu}_{2} \mathrm{~V}_{2} \mathrm{O}_{7}$, Phys. Rev. B 92, 024423 (2015).

[66] C. Darie, C. Goujon, M. Bacia, H. Klein, P. Toulemonde, P. Bordet, and E. Suard, Magnetic and Crystal Structures of $\mathrm{BiCrO}_{3}$, Solid State Sci. 12, 660 (2010).

[67] P. Schobinger-Papamantellos, K. H. Buschow, and J. Rodríguez-Carvajal, Magnetoelastic Phase Transitions in the $\mathrm{LuFe}_{4} \mathrm{Ge}_{2}$ and $\mathrm{YFe}_{4} \mathrm{Si}_{2}$ Compounds: A Neutron Diffraction Study, J. Magn. Magn. Mater. 324, 3709 (2012).

[68] P. Schobinger-Papamantellos, D. De Mooij, and K. Buschow, Crystallographic and Magnetic Structure of $\mathrm{TbGe}_{2}$, J. Less-Common Met. 144, 265 (1988).

[69] W. Kunnmann, S. La Placa, L. Corliss, J. Hastings, and E. Banks, Magnetic Structures of the Ordered Trirutiles $\mathrm{Cr}_{2} \mathrm{WO}_{6}, \mathrm{Cr}_{2} \mathrm{TeO}_{6}$ and $\mathrm{Fe}_{2} \mathrm{TeO}_{6}$, J. Phys. Chem. Solids 29, 1359 (1968).

[70] M. Avdeev, B. J. Kennedy, and T. Kolodiazhnyi, Neutron Powder Diffraction Study of the Magnetic Structure of $\mathrm{EuZrO}_{3}$, J. Phys. Condens. Matter 26, 095401 (2014).

[71] P. D. Battle, C. P. Grey, M. Hervieu, C. Martin, C. A. Moore, and Y. Paik, Structural Chemistry and Magnetic Properties of $\mathrm{La}_{2} \mathrm{LiRuO}_{6}$, J. Solid State Chem. 175, 20 (2003).

[72] D. I. Gorbunov, M. S. Henriques, A. V. Andreev, V. Eigner, A. Gukasov, X. Fabrèges, Y. Skourski, V. Petříček, and J. Wosnitza, Magnetic Anisotropy and Reduced Neodymium Magnetic Moments in $\mathrm{Nd}_{3} \mathrm{Ru}_{4} \mathrm{Al}_{1}$ 2, Phys. Rev. B 93, 024407 (2016).

[73] Z. Yamani, Z. Tun, and D. H. Ryan, Neutron Scattering Study of the Classical Antiferromagnet $\mathrm{MnF}_{2}$ : A Perfect Hands-On Neutron Scattering Teaching Course, Special Issue on Neutron Scattering in Canada, Can. J. Phys. 88, 771 (2010).

[74] S. Yano, D. Louca, J. Yang, U. Chatterjee, D. E. Bugaris, D. Y. Chung, L. Peng, M. Grayson, and M. G. Kanatzidis, Magnetic Structure of $\mathrm{NiS}_{2-x} \mathrm{Se}_{x}$, Phys. Rev. B 93, 024409 (2016).

[75] N. Taira, M. Wakeshima, Y. Hinatsu, A. Tobo, and K. Ohoyama, Magnetic Structure of Pyrochlore-Type $\mathrm{Er}_{2} \mathrm{Ru}_{2} \mathrm{O}_{7}$, J. Solid State Chem. 176, 165 (2003).

[76] L. Ding, C. V. Colin, C. Darie, J. Robert, F. Gay, and P. Bordet, One-Dimensional Short-Range Magnetic Correlations in the Magnetoelectric Pyroxene $\mathrm{CaMnGe}_{2} \mathrm{O}_{6}$, Phys. Rev. B 93, 064423 (2016).

[77] G. J. Redhammer, G. Roth, W. Treutmann, W. Paulus, G. André, C. Pietzonka, and G. Amthauer, Magnetic Ordering and Spin Structure in Ca-Bearing Clinopyroxenes $\mathrm{Ca}^{2+}(\mathrm{Si}, \mathrm{Ge})_{2} \mathrm{O}_{6}, M=\mathrm{Fe}, \mathrm{Ni}, \mathrm{Co}, \mathrm{Mn}$, J. Solid State Chem. 181, 3163 (2008).

[78] A. Yaouanc, P. D. de Réotier, P. Bonville, J. A. Hodges, V. Glazkov, L. Keller, V. Sikolenko, M. Bartkowiak, A. Amato, C. Baines, P. J. C. King, P. C. M. Gubbens, and A. Forget, Dynamical Splayed Ferromagnetic Ground State in the Quantum Spin Ice $\mathrm{Yb}_{2} \mathrm{Sn}_{2} \mathrm{O}_{7}$, Phys. Rev. Lett. 110, 127207 (2013).

[79] J. Gaudet, K. A. Ross, E. Kermarrec, N. P. Butch, G. Ehlers, H. A. Dabkowska, and B. D. Gaulin, Gapless Quantum Excitations from an Icelike Splayed Ferromagnetic Ground 
State in Stoichiometric $\mathrm{Yb}_{2} \mathrm{Ti}_{2} \mathrm{O}_{7}$, Phys. Rev. B 93, 064406 (2016).

[80] K. Knížek, Z. Jirák, P. Novák, and C. de la Cruz, NonCollinear Magnetic Structures of $\mathrm{TbCoO}_{3}$ and $\mathrm{DyCoO}_{3}$, Solid State Sci. 28, 26 (2014).

[81] V. Scagnoli, M. Allieta, H. Walker, M. Scavini, T. Katsufuji, L. Sagarna, O. Zaharko, and C. Mazzoli, EuTiO 3 Magnetic Structure Studied by Neutron Powder Diffraction and Resonant X-Ray Scattering, Phys. Rev. B 86, 094432 (2012).

[82] E. Ressouche, M. Loire, V. Simonet, R. Ballou, A. Stunault, and A. Wildes, Magnetoelectric $\mathrm{MnPS}_{3}$ as a Candidate for Ferrotoroidicity, Phys. Rev. B 82, 100408 (R) (2010).

[83] J. Blasco, J. García, G. Subías, J. Stankiewicz, J. A. Rodríguez-Velamazán, C. Ritter, J. L. García-Muñoz, and F. Fauth, Magnetoelectric and Structural Properties of $\mathrm{Y}_{2} \mathrm{CoMnO}_{6}$ : The Role of Antisite Defects, Phys. Rev. B 93, 214401 (2016).

[84] L. D. Sanjeewa, V. O. Garlea, M. A. McGuire, C. D. McMillen, H. Cao, and J. W. Kolis, Structural and Magnetic Characterization of the One-Dimensional $S=\frac{5}{2}$ Antiferromagnetic Chain System $\operatorname{SrMn}\left(\mathrm{VO}_{4}\right)(\mathrm{OH})$, Phys. Rev. B 93, 224407 (2016).

[85] A. Scheie, M. Sanders, J. Krizan, Y. Qiu, R. J. Cava, and C. Broholm, Effective Spin- $\frac{1}{2}$ Scalar Chiral Order on Kagome Lattices in $\mathrm{Nd}_{3} \mathrm{Sb}_{3} \mathrm{Mg}_{2} \mathrm{O}_{14}$, Phys. Rev. B 93, 180407(R) (2016).

[86] G. Ferey, M. Leblanc, R. De Pape, and J. Pannetier, Frustrated Magnetic Structures: II. Antiferromagnetic Structure of the Ordered Modified Pyrochlore $\mathrm{NH}_{4} \mathrm{Fe}^{I I} \mathrm{Fe}^{I I I} \mathrm{~F}_{6}$ at $4.2 \sim K$, Solid State Commun. 53, 559 (1985).

[87] P. Burlet, J. Rossat-Mignod, R. Troć, and Z. Henkie, NonColinear Magnetic Structure of $\mathrm{U}_{3} \mathrm{P}_{4}$ and $\mathrm{U}_{3} \mathrm{As}_{4}$, Solid State Commun. 39, 745 (1981).

[88] G. Rousse, J. Rodriguez-Carvajal, S. Patoux, and C. Masquelier, Magnetic Structures of the Triphylite $\mathrm{LiFePO}_{4}$ and of Its Delithiated Form $\mathrm{FePO}_{4}$, Chem. Mater. 15, 4082 (2003).

[89] L. S. Wu, S. E. Nikitin, M. Frontzek, A. I. Kolesnikov, G. Ehlers, M. D. Lumsden, K. A. Shaykhutdinov, E.-J. Guo, A. T. Savici, Z. Gai, A.S. Sefat, and A. Podlesnyak, Magnetic Ground State of the Ising-like Antiferromagnet $\mathrm{DyScO}_{3}$, Phys. Rev. B 96, 144407 (2017).

[90] M. S. Henriques, D. I. Gorbunov, A. V. Andreev, X. Fabrèges, A. Gukasov, M. Uhlarz, V. Petríček, B. Ouladdiaf, and J. Wosnitza, Complex Magnetic Order in the Kagome Ferromagnet $\operatorname{Pr}_{3} \mathrm{Ru}_{4} \mathrm{Al}_{12}$, Phys. Rev. B 97, 014431 (2018).

[91] D. Fruchart and E. F. Bertaut, Magnetic Studies of the Metallic Perovskite-Type Compounds of Manganese, J. Phys. Soc. Jpn. 44, 781 (1978).

[92] W. Jauch, M. Reehuis, and A. J. Schultz, $\gamma$-Ray and Neutron Diffraction Studies of $\mathrm{CoF}_{2}$ : Magnetostriction, Electron Density and Magnetic Moments, Acta Crystallogr. Sect. A 60, 51 (2004).

[93] Y. Singh, M. A. Green, Q. Huang, A. Kreyssig, R. J. McQueeney, D. C. Johnston, and A. I. Goldman, Magnetic
Order in $\mathrm{BaMn}_{2} \mathrm{As}_{2}$ from Neutron Diffraction Measurements, Phys. Rev. B 80, 100403(R) (2009).

[94] A. Wiedenmann, J. Rossat-Mignod, A. Louisy, R. Brec, and J. Rouxel, Neutron Diffraction Study of the Layered Compounds $\mathrm{MnPSe}_{3}$ and $\mathrm{FePSe}_{3}$, Solid State Commun. 40, 1067 (1981).

[95] Q. Zhang, W. Tian, S. G. Peterson, K. W. Dennis, and D. Vaknin, Spin Reorientation and Ce-Mn Coupling in Antiferromagnetic Oxypnictide CeMnAsO, Phys. Rev. B 91, 064418 (2015).

[96] D. Xu, M. Avdeev, P. D. Battle, and X.-Q. Liu, Magnetic Properties of $\mathrm{CeMn}_{2-x} \mathrm{Co}_{x} \mathrm{Ge}_{4} \mathrm{O}_{12}(0 \leq x \leq 2)$ as a Function of Temperature and Magnetic Field, Inorg. Chem. 56, 2750 (2017).

[97] G. Shirane, S. J. Pickart, and Y. Ishikawa, Neutron Diffraction Study of Antiferromagnetic $\mathrm{MnTiO}_{3}$ and $\mathrm{NiTiO}_{3}$, J. Phys. Soc. Jpn. 14, 1352 (1959).

[98] A. C. Garcia-Castro, W. Ibarra-Hernandez, E. Bousquet, and A.H. Romero, Direct Magnetization-Polarization Coupling in $\mathrm{BaCuF}_{4}$, Phys. Rev. Lett. 121, 117601 (2018).

[99] S. W. Kim, S.-H. Kim, P. S. Halasyamani, M. A. Green, K. P. Bhatti, C. Leighton, H. Das, and C. J. Fennie, $\mathrm{RbFe}^{2+} \mathrm{Fe}^{3+} \mathrm{F}_{6}$ : Synthesis, Structure, and Characterization of a New Charge-Ordered Magnetically Frustrated Pyrochlore-Related Mixed-Metal Fluoride, Chem. Sci. 3, 741 (2012).

[100] R. Santoro, D. Segal, and R. Newnham, Magnetic Properties of $\mathrm{LiCoPO}_{4}$ and $\mathrm{LiNiPO}_{4}$, J. Phys. Chem. Solids 27 , 1192 (1966).

[101] R. A. Steeman, E. Frikkee, S. A. M. Mentink, A. A. Menovsky, G. J. Nieuwenhuys, and J. A. Mydosh, Hybridisation Effects in $\mathrm{UPt}_{2} \mathrm{Si}_{2}$, J. Phys. Condens. Matter 2, 4059 (1990).

[102] E. Palacios, M. Evangelisti, R. Sáez-Puche, A. J. Dos Santos-García, F. Fernández-Martínez, C. Cascales, M. Castro, R. Burriel, O. Fabelo, and J. A. RodríguezVelamazán, Magnetic Structures and Magnetocaloric Effect in $R \mathrm{VO}_{4}(R=\mathrm{Gd}, \mathrm{Nd})$, Phys. Rev. B 97, 214401 (2018).

[103] P. J. Brown, V. Nunezt, F. Tassett, J. B. Forsytht, and P. Radhakrishnao, Determination of the Magnetic Structure of Mn, Sn Using Generalized Neutron Polarization Analysis, J. Phys. Condens. Matter 2, 9409 (1990).

[104] P. Burlet, E. Ressouche, B. Malaman, R. Welter, J. P. Sanchez, and P. Vulliet, Noncollinear Magnetic Structure of $\mathrm{MnTe}_{2}$, Phys. Rev. B 56, 14013 (1997).

[105] A. S. Sukhanov, S. Singh, L. Caron, T. Hansen, A. Hoser, V. Kumar, H. Borrmann, A. Fitch, P. Devi, K. Manna, C. Felser, and D.S. Inosov, Gradual Pressure-Induced Change in the Magnetic Structure of the Noncollinear Antiferromagnet $\mathrm{Mn}_{3}$ Ge, Phys. Rev. B 97, 214402 (2018).

[106] A. S. Cavichini, M. T. Orlando, J. B. Depianti, J. L. Passamai, F. Damay, F. Porcher, and E. Granado, Exotic Magnetism and Spin-Orbit-Assisted Mott Insulating State in a 3d-5d Double Perovskite, Phys. Rev. B 97, 054431 (2018).

[107] X. F. Hao, A. Stroppa, S. Picozzi, A. Filippetti, and C. Franchini, Exceptionally Large Room-Temperature Ferroelectric Polarization in the $\mathrm{PbNiO}_{3}$ Multiferroic Nickelate: First-Principles Study, Phys. Rev. B 86, 014116 (2012). 
[108] C. Autret, C. Martin, M. Hervieu, R. Retoux, B. Raveau, G. André, and F. Bourée, Structural Investigation of $\mathrm{Ca}_{2} \mathrm{MnO}_{4}$ by Neutron Powder Diffraction and Electron Microscopy, J. Solid State Chem. 177, 2044 (2004).

[109] S. L. Brock, N. Raju, J. Greedan, and S. M. Kauzlarich, The Magnetic Structures of the Mixed Layer Pnictide Oxide Compounds $\mathrm{Sr}_{2} \mathrm{Mn}_{3} \mathrm{Pn}_{2} \mathrm{O}_{2}(\mathrm{Pn}=\mathrm{As}, \mathrm{Sb})$, J. Alloys Compd. 237, 9 (1996).

[110] L. Regnault, J. Henry, J. Rossat-Mignod, and A. De Combarieu, Magnetic Properties of the Layered Nickel Compounds $\mathrm{BaNi}_{2}\left(\mathrm{PO}_{4}\right)_{2}$ and $\mathrm{BaNi}_{2}\left(\mathrm{AsO}_{4}\right)_{2}$, J. Magn. Magn. Mater. 15-18, 1021 (1980).

[111] A. Sazonov, M. Meven, V. Hutanu, G. Heger, T. Hansen, and A. Gukasov, Magnetic Behaviour of Synthetic $\mathrm{Co}_{2} \mathrm{SiO}_{4}$, Acta Crystallogr. Sect. B 65, 664 (2009).

[112] G. Will and W. Schafer, Neutron Diffraction and the Magnetic Structures of Some Rare Earth Diborides and Tetraborides, J. Less-Common Met. 67, 31 (1979).

[113] W. Lottermoser and H. Fuess, Magnetic Structure of the Orthosilicates $\mathrm{Mn}_{2} \mathrm{SiO}_{4}$ and $\mathrm{Co}_{2} \mathrm{SiO}_{4}$, Phys. Status Solidi A 109, 589 (1988).

[114] W. Lottermoser, R. Müller, and H. Fuess, Antiferromagnetism in Synthetic Olivines, J. Magn. Magn. Mater. 54-57, 1005 (1986).

[115] P. Wadley, V. Hills, M. R. Shahedkhah, K. W. Edmonds, R. P. Campion, V. Novák, B. Ouladdiaf, D. Khalyavin, S. Langridge, V. Saidl, P. Nemec, A. W. Rushforth, B. L. Gallagher, S. S. Dhesi, F. Maccherozzi, J. Železný, and T. Jungwirth, Antiferromagnetic Structure in Tetragonal CuMnAs Thin Films, Sci. Rep. 5, 17079 (2015).

[116] Z. Ouyang, F. Wang, Q. Hang, W. Liu, G. Liu, J. Lynn, J. Liang, and G. Rao, Temperature Dependent Neutron Powder Diffraction Study of the Laves Phase Compound $\mathrm{TbCo}_{2}$, J. Alloys Compd. 390, 21 (2005).

[117] M. Sale, Q. Xia, M. Avdeev, and C. D. Ling, Crystal and Magnetic Structures of Melilite-Type $\mathrm{Ba}_{2} \mathrm{MnSi}_{2} \mathrm{O}_{7}$, Inorg. Chem. 58, 4164 (2019).

[118] M. V. Lobanov, M. Greenblatt, E. a. N. Caspi, J. D. Jorgensen, D. V. Sheptyakov, B. H. Toby, C. E. Botez, and P. W. Stephens, Crystal and Magnetic Structure of the $\mathrm{Ca}_{3} \mathrm{Mn}_{2} \mathrm{O}_{7}$ Ruddlesden-Popper Phase: Neutron and Synchrotron X-Ray Diffraction Study, J. Phys. Condens. Matter 16, 5339 (2004).

[119] M. Sale, M. Avdeev, Z. Mohamed, C. D. Ling, and P. Barpanda, Magnetic Structure and Properties of Centrosymmetric Twisted-Melilite $\mathrm{K}_{2} \mathrm{CoP}_{2} \mathrm{O}_{7}$, Dalton Trans. 46, 6409 (2017).

[120] E. Solana-Madruga, Á. M. Arévalo-López, A. J. Dos santos-García, C. Ritter, C. Cascales, R. Sáez-Puche, and J. P. Attfield, Anisotropic Magnetic Structures of the Mn $\mathrm{RMnSbO}_{6}$ High-Pressure Doubly Ordered Perovskites $(R=\mathrm{La}, \operatorname{Pr}$, and Nd), Phys. Rev. B 97, 134408 (2018).

[121] V. Gvozdetskyi, A. Senyshyn, R. Gladyshevskii, and V. Hlukhyy, Crystal and Magnetic Structures of the Chain Antiferromagnet $\mathrm{CaFe}_{4} \mathrm{Al}_{8}$, Inorg. Chem. 57, 5820 (2018).

[122] S. Petit, E. Lhotel, F. Damay, P. Boutrouille, A. Forget, and D. Colson, Long-Range Order in the Dipolar XY Antiferromagnet $\mathrm{Er}_{2} \mathrm{Sn}_{2} \mathrm{O}_{7}$, Phys. Rev. Lett. 119, 187202 (2017).
[123] A. M. Hallas, J. Gaudet, N. P. Butch, G. Xu, M. Tachibana, C. R. Wiebe, G. M. Luke, and B. D. Gaulin, Phase Competition in the Palmer-Chalker XY Pyrochlore $\mathrm{Er}_{2} \mathrm{Pt}_{2} \mathrm{O}_{7}$, Phys. Rev. Lett. 119, 187201 (2017).

[124] S. Calder, D. J. Singh, V. O. Garlea, M. D. Lumsden, Y. G. Shi, K. Yamaura, and A. D. Christianson, Interplay of Spin-Orbit Coupling and Hybridization in $\mathrm{Ca}_{3} \mathrm{LiOsO}_{6}$ and $\mathrm{Ca}_{3} \mathrm{LiRuO}_{6}$, Phys. Rev. B 96, 184426 (2017).

[125] R. Toft-Petersen, N. H. Andersen, H. Li, J. Li, W. Tian, S. L. Bud'ko, T. B. S. Jensen, C. Niedermayer, M. Laver, O. Zaharko, J. W. Lynn, and D. Vaknin, Magnetic Phase Diagram of Magnetoelectric $\mathrm{LiMnPO}_{4}$, Phys. Rev. B 85, 224415 (2012).

[126] S. Calder, V. O. Garlea, D. F. McMorrow, M. D. Lumsden, M. B. Stone, J. C. Lang, J.-W. Kim, J. A. Schlueter, Y. G. Shi, K. Yamaura, Y.S. Sun, Y. Tsujimoto, and A. D. Christianson, Magnetically Driven Metal-Insulator Transition in $\mathrm{NaOsO}_{3}$, Phys. Rev. Lett. 108, 257209 (2012).

[127] S. Baran, D. Kaczorowski, A. Arulraj, B. Penc, and A. Szytuła, Frustrated Magnetic Structure of TmAgGe, J. Magn. Magn. Mater. 321, 3256 (2009).

[128] P. Schobinger-Papamantellos, J. Rodriguez-Carvajal, G. André, N. Duong, K. Buschow, and P. Tolédano, Simultaneous Structural and Magnetic Transitions in $\mathrm{YFe}_{4} \mathrm{Ge}_{2}$ Studied by Neutron Diffraction and Magnetic Measurements, J. Magn. Magn. Mater. 236, 14 (2001).

[129] G. J. Redhammer, G. Roth, W. Treutmann, M. Hoelzel, W. Paulus, G. André, C. Pietzonka, and G. Amthauer, The Magnetic Structure of Clinopyroxene-Type $\mathrm{LiFeGe}_{2} \mathrm{O}_{6}$ and Revised Data on Multiferroic $\mathrm{LiFeSi}_{2} \mathrm{O}_{6}$, J. Solid State Chem. 182, 2374 (2009).

[130] R. Trump, S. Thierfeldt, M. Loewenhaupt, and T. Chattopadhyay, Magnetic Structure of the Kondo Lattice Compound $\mathrm{CeCu}_{2}$, J. Appl. Phys. 69, 4699 (1991).

[131] S. Calder, M. D. Lumsden, V. O. Garlea, J. W. Kim, Y. G. Shi, H. L. Feng, K. Yamaura, and A. D. Christianson, Magnetic Structure Determination of $\mathrm{Ca}_{3} \mathrm{LiOsO}_{6}$ Using Neutron and X-Ray Scattering, Phys. Rev. B 86, 054403 (2012).

[132] X. Fabrèges, I. Mirebeau, P. Bonville, S. Petit, G. LebrasJasmin, A. Forget, G. André, and S. Pailhès, Magnetic Order in $\mathrm{YbMnO}_{3}$ Studied by Neutron Diffraction and Mössbauer Spectroscopy, Phys. Rev. B 78, 214422 (2008).

[133] J. Blasco, J. L. García-Muñoz, J. García, G. Subías, J. Stankiewicz, J. A. Rodríguez-Velamazán, and C. Ritter, Magnetic Order and Magnetoelectric Properties of $R_{2} \mathrm{CoMnO}_{6}$ Perovskites ( $R=\mathrm{Ho}, \mathrm{Tm}, \mathrm{Yb}$, and $\mathrm{Lu}$ ), Phys. Rev. B 96, 024409 (2017).

[134] P. J. Brown and T. Chatterji, Neutron Diffraction and Polarimetric Study of the Magnetic and Crystal Structures of $\mathrm{HoMnO}_{3}$ and $\mathrm{YMnO}_{3}$, J. Phys. Condens. Matter 18, 10085 (2006).

[135] P. J. Brown and J. B. Forsyth, A Neutron Diffraction Study of Weak Ferromagnetism in Nickel Fluoride, J. Phys. C 14, 5171 (1981).

[136] A. G. Gukasov, P. Rogl, P. J. Brown, M. Mihalik, and A. Menovsky, Site Susceptibility Tensors and Magnetic Structure of $\mathrm{U}_{3} \mathrm{Al}_{2} \mathrm{Si}_{3}$ : A Polarized Neutron Diffraction Study, J. Phys. Condens. Matter 14, 8841 (2002). 
[137] A. A. Aczel, D. E. Bugaris, J. Yeon, C. de la Cruz, H.-C. zur Loye, and S. E. Nagler, Coupled $N d$ and $B^{\prime}$ Spin Ordering in the Double Perovskites $\mathrm{Nd}_{2} \mathrm{Na}^{\prime} \mathrm{O}_{6}\left(B^{\prime}=\mathrm{Ru}\right.$, Os), Phys. Rev. B 88, 014413 (2013).

[138] V. Nguyen, F. Tchéou, and J. Rossat-Mignod, Magnetic Structures of PrSi and NdSi Intermetallic Alloys, Solid State Commun. 23, 821 (1977).

[139] D. Gignoux, J. Rossat-Mignod, and F. Tcheou, Magnetic Structure of the TmNi Compound Crystal Field Effect, Phys. Status Solidi A 14, 483 (1972).

[140] J. Rodriguez-Carvajal, M. T. Fernandez-Diaz, and J. L. Martinez, Neutron Diffraction Study on Structural and Magnetic Properties of $\mathrm{La}_{2} \mathrm{NiO}_{4}$, J. Phys. Condens. Matter 3, 3215 (1991).

[141] K. Tomiyasu and I. Kagomiya, Magnetic Structure of $\mathrm{NiCr}_{2} \mathrm{O}_{4}$ Studied by Neutron Scattering and Magnetization Measurements, J. Phys. Soc. Jpn. 73, 2539 (2004).

[142] E. Bertaut, J. Cohen, B. Lambert-Andron, and P. Mollard, Étude de $\mathrm{Cr}_{2} \mathrm{~S}_{3}$ Rhomboédrique par Diffraction Neutronique et Mesures Magnétiques, J. Phys. (Paris) 29, 813 (1968).

[143] A. M. Arévalo-López and J. P. Attfield, Weak Ferromagnetism and Domain Effects in Multiferroic $\mathrm{LiNbO}_{3}$-Type $\mathrm{MnTiO}_{3}$-II, Phys. Rev. B 88, 104416 (2013).

[144] V. Hutanu, A. Sazonov, M. Meven, H. Murakawa, Y. Tokura, S. Bordács, I. Kézsmárki, and B. Náfrádi, Determination of the Magnetic Order and the Crystal Symmetry in the Multiferroic Ground State of $\mathrm{Ba}_{2} \mathrm{CoGe}_{2} \mathrm{O}_{7}$, Phys. Rev. B 86, 104401 (2012).

[145] B. Roy, A. Pandey, Q. Zhang, T. W. Heitmann, D. Vaknin, D. C. Johnston, and Y. Furukawa, Experimental Evidence of a Collinear Antiferromagnetic Ordering in the Frustrated $\mathrm{CoAl}_{2} \mathrm{O}_{4}$ Spinel, Phys. Rev. B 88, 174415 (2013).

[146] A. Muñoz, J. A. Alonso, M. J. Martínez-Lope, M. T. Casáis, J. L. Martínez, and M. T. Fernández-Díaz, Magnetic Structure of Hexagonal $R \mathrm{MnO}_{3}(R=\mathrm{Y}, \mathrm{Sc})$ : Thermal Evolution from Neutron Powder Diffraction Data, Phys. Rev. B 62, 9498 (2000).

[147] V. O. Garlea, R. Jin, D. Mandrus, B. Roessli, Q. Huang, M. Miller, A. J. Schultz, and S. E. Nagler, Magnetic and Orbital Ordering in the Spinel $\mathrm{MnV}_{2} \mathrm{O}_{4}$, Phys. Rev. Lett. 100, 066404 (2008).

[148] A. H. Hill, F. Jiao, P. G. Bruce, A. Harrison, W. Kockelmann, and C. Ritter, Neutron Diffraction Study of Mesoporous and Bulk Hematite, $\alpha-\mathrm{Fe}_{2} \mathrm{O}_{3}$, Chem. Mater. 20, 4891 (2008).

[149] Y. F. Guo, A. J. Princep, X. Zhang, P. Manuel, D. Khalyavin, I. I. Mazin, Y. G. Shi, and A. T. Boothroyd, Coupling of Magnetic Order to Planar Bi Electrons in the Anisotropic Dirac Metals $A \mathrm{MnBi}_{2}(A=\mathrm{Sr}, C a)$, Phys. Rev. B 90, 075120 (2014).

[150] S. Iikubo, K. Kodama, K. Takenaka, H. Takagi, and S. Shamoto, Magnetovolume Effect in $\mathrm{Mn}_{3} \mathrm{Cu}_{1-x} \mathrm{Ge}_{x} \mathrm{~N}$ Related to the Magnetic Structure: Neutron Powder Diffraction Measurements, Phys. Rev. B 77, 020409(R) (2008).

[151] M. Zhu, D. Do, C. R. Dela Cruz, Z. Dun, H. D. Zhou, S. D. Mahanti, and X. Ke, Tuning the Magnetic Exchange via a Control of Orbital Hybridization in $\mathrm{Cr}_{2}\left(\mathrm{Te}_{1-x} \mathrm{~W}_{x}\right) \mathrm{O}_{6}$, Phys. Rev. Lett. 113, 076406 (2014).
[152] O. S. Volkova, V. V. Mazurenko, I. V. Solovyev, E. B. Deeva, I. V. Morozov, J.-Y. Lin, C. K. Wen, J. M. Chen, M. Abdel-Hafiez, and A. N. Vasiliev, Noncollinear Ferrimagnetic Ground State in $\mathrm{Ni}\left(\mathrm{NO}_{3}\right)_{2}$, Phys. Rev. B 90 , 134407 (2014).

[153] A. P. Sazonov, A. Gukasov, H. B. Cao, P. Bonville, E. Ressouche, C. Decorse, and I. Mirebeau, Magnetic Structure in the Spin Liquid $\mathrm{Tb}_{2} \mathrm{Ti}_{2} \mathrm{O}_{7}$ Induced by a [111] Magnetic Field: Search for a Magnetization Plateau, Phys. Rev. B 88, 184428(R) (2013).

[154] K. Ohgushi, J.-i. Yamaura, H. Ohsumi, K. Sugimoto, S. Takeshita, A. Tokuda, H. Takagi, M. Takata, and T.-h. Arima, Resonant X-Ray Diffraction Study of the Strongly Spin-Orbit-Coupled Mott Insulator $\mathrm{CaIrO}_{3}$, Phys. Rev. Lett. 110, 217212 (2013).

[155] A. Purwanto, R. A. Robinson, L. Havela, V. Sechovský, P. Svoboda, H. Nakotte, K. Prokeš, F. R. de Boer, A. Seret, J. M. Winand, J. Rebizant, and J. C. Spirlet, Magnetic Ordering in $\mathrm{U}_{2} \mathrm{Pd}_{2} \mathrm{In}$ and $\mathrm{U}_{2} \mathrm{Pd}_{2} \mathrm{Sn}$, Phys. Rev. B 50, 6792 (1994).

[156] T. B. S. Jensen, N. B. Christensen, M. Kenzelmann, H. M. Rønnow, C. Niedermayer, N. H. Andersen, K. Lefmann, J. Schefer, M. v. Zimmermann, J. Li, J. L. Zarestky, and D. Vaknin, Field-Induced Magnetic Phases and Electric Polarization in $\mathrm{LiNiPO}_{4}$, Phys. Rev. B 79, 092412 (2009).

[157] S. Calder, B. Saparov, H. B. Cao, J. L. Niedziela, M. D. Lumsden, A. S. Sefat, and A. D. Christianson, Magnetic Structure and Spin Excitations in $\mathrm{BaMn}_{2} \mathrm{Bi}_{2}$, Phys. Rev. B 89, 064417 (2014).

[158] J. A. Blanco, P. J. Brown, A. Stunault, K. Katsumata, F. Iga, and S. Michimura, Magnetic Structure of $\mathrm{GdB}_{4}$ from Spherical Neutron Polarimetry, Phys. Rev. B 73, 212411 (2006).

[159] P. J. Brown and B.C. Frazer, Magnetic Structure of $\mathrm{CoSO}_{4}$, Phys. Rev. 129, 1145 (1963).

[160] J. A. Gonzalo, D. E. Cox, and G. Shirane, The Magnetic Structure of $\mathrm{FeSb}_{2} \mathrm{O}_{4}$, Phys. Rev. 147, 415 (1966).

[161] B. Campbell, J. M. Perez-Mato, V. Petricek, J. RodriguezCarvajal, and W. Sikora, Magnetic CIF Dictionary (2020), https://www.iucr.org/resources/cif/dictionaries.

[162] L. D. Landau and E. M. Lifshitz, Course of Theoretical Physics (Elsevier, New York, 2013).

[163] M. I. Aroyo, J. M. Perez-Mato, D. Orobengoa, E. Tasci, G. De La Flor, and A. Kirov, Crystallography Online: Bilbao Crystallographic Server, Bulg Chem Commun 43, 183 (2011).

[164] M. B. Wilk and R. Gnanadesikan, Probability Plotting Methods for the Analysis for the Analysis of Data, Biometrika 55, 1 (1968).

[165] S. F. A. Kettle, Physical Inorganic Chemistry: A Coordination Chemistry Approach (Springer, Berlin, 2013), pp. 1-503.

[166] M. El-Batanouny, Advanced Quantum Condensed Matter Physics (Cambridge University Press, Cambridge, England, 2020), https://doi.org/10.1017/9781108691291.

[167] The spin-orbit coupling is proportional to $d V / d r$, where $V$ is the potential due to the ions. Hence, heavier elements exhibit stronger spin-orbit coupling. See, e.g., Ref. [8].

[168] For instance, the $3+$ ion for $\operatorname{Er}$ has $4 f^{11}$ and thus 3 unpaired spins yielding $s=3 / 2$. The orbital angular 
momentum is maximized when orbitals with magnetic quantum number $m_{l}=3,2,1$ are singly occupied yielding $l=6$ and $L=I$. Finally the total angular momentum $\boldsymbol{J}=\boldsymbol{S}+\boldsymbol{L}$ for more than half filling, i.e., quantum number $j=15 / 2$. The ground state term symbol reads ${ }^{4} I_{15 / 2}$.

[169] O. P. Vajk, M. Kenzelmann, J. W. Lynn, S. B. Kim, and S.-W. Cheong, Magnetic Order and Spin Dynamics in Ferroelectric $\mathrm{HoMnO}_{3}$, Phys. Rev. Lett. 94, 087601 (2005).

[170] T. Lottermoser, T. Lonkai, U. Amann, D. Hohlwein, J. Ihringer, and M. Fiebig, Magnetic Phase Control by an Electric Field, Nature (London) 430, 541 (2004).

[171] A. Muñoz, J. A. Alonso, M. J. Martínez-Lope, M. T. Casáis, J. L. Martínez, and M. T. Fernández-Díaz, Evolution of the Magnetic Structure of Hexagonal $\mathrm{HoMnO}_{3}$ from Neutron Powder Diffraction Data, Chem. Mater. 13, 1497 (2001).

[172] M. Fiebig, D. Fröhlich, K. Kohn, S. Leute, T. Lottermoser, V. V. Pavlov, and R. V. Pisarev, Determination of the Magnetic Symmetry of Hexagonal Manganites by Second Harmonic Generation, Phys. Rev. Lett. 84, 5620 (2000).

[173] V. I. Anisimov, J. Zaanen, and O. K. Andersen, Band Theory and Mott Insulators: Hubbard U Instead of Stoner I, Phys. Rev. B 44, 943 (1991).

[174] A. Georges, G. Kotliar, W. Krauth, and M. J. Rozenberg, Dynamical Mean-Field Theory of Strongly Correlated Fermion Systems and the Limit of Infinite Dimensions, Rev. Mod. Phys. 68, 13 (1996).

[175] G. Kotliar and D. Vollhardt, Strongly Correlated Materials: Insights from Dynamical Mean-Field Theory, Phys. Today 57, No. 3, 53 (2004).

[176] V. Anisimov, A. Poteryaev, M. Korotin, A. Anokhin, and G. Kotliar, First-Principles Calculations of the Electronic Structure and Spectra of Strongly Correlated Systems: Dynamical Mean-Field Theory, J. Phys. Condens. Matter 9, 7359 (1997).

[177] A. I. Lichtenstein and M. I. Katsnelson, Ab Initio Calculations of Quasiparticle Band Structure in Correlated Systems: LDA ${ }^{++}$Approach, Phys. Rev. B 57, 6884 (1998).

[178] K. Held, I. Nekrasov, G. Keller, V. Eyert, N. Blümer, A. McMahan, R. Scalettar, T. Pruschke, V. Anisimov, and D. Vollhardt, Realistic Investigations of Correlated Electron Systems with LDA + DMFT, Phys. Status Solidi B 243, 2599 (2006).

[179] K. Held, O. Andersen, M. Feldbacher, A. Yamasaki, and Y. Yang, Bandstructure Meets Many-Body Theory: The LDA + DMFT Method, J. Phys. Condens. Matter 20, 064202 (2008).

[180] J. Kaufmann, C. Eckhardt, M. Pickem, M. Kitatani, A. Kauch, and K. Held, Self-Consistent Ab Initio dra Approach, arXiv:2010.03938 [Phys. Rev. B (to be published)].

[181] E. Gull, A. J. Millis, A. I. Lichtenstein, A. N. Rubtsov, M. Troyer, and P. Werner, Continuous-Time Monte Carlo Methods for Quantum Impurity Models, Rev. Mod. Phys. 83, 349 (2011).

[182] G. Rohringer, H. Hafermann, A. Toschi, A. A. Katanin, A. E. Antipov, M. I. Katsnelson, A. I. Lichtenstein, A. N. Rubtsov, and K. Held, Diagrammatic Routes to Nonlocal
Correlations beyond Dynamical Mean Field Theory, Rev. Mod. Phys. 90, 025003 (2018).

[183] J. M. An, S. V. Barabash, V. Ozolins, M. van Schilfgaarde, and K. D. Belashchenko, First-Principles Study of Phase Stability of Gd-Doped EuO and EuS, Phys. Rev. B 83, 064105 (2011).

[184] M. Schlipf, M. Betzinger, M. Ležaić, C. Friedrich, and S. Blügel, Structural, Electronic, and Magnetic Properties of the Europium Chalcogenides: A Hybrid-Functional DFT Study, Phys. Rev. B 88, 094433 (2013).

[185] R. Kaneko, M.-T. Huebsch, S. Sakai, R. Arita, H. Shinaoka, K. Ueda, Y. Tokura, and J. Fujioka, Enhanced Thermopower in the Correlated Semimetallic Phase of Hole-Doped Pyrochlore Iridates, Phys. Rev. B 99, 161104 (R) (2019).

[186] A. Pulkkinen, B. Barbiellini, J. Nokelainen, V. Sokolovskiy, D. Baigutlin, O. Miroshkina, M. Zagrebin, V. Buchelnikov, C. Lane, R. S. Markiewicz et al., Coulomb Correlation in Noncollinear Antiferromagnetic $\alpha$-Mn, Phys. Rev. B 101, 075115 (2020).

[187] M. Cococcioni and S. de Gironcoli, Linear Response Approach to the Calculation of the Effective Interaction Parameters in the LDA + U Method, Phys. Rev. B 71, 035105 (2005).

[188] E. Şaş1oğlu, C. Friedrich, and S. Blügel, Effective Coulomb Interaction in Transition Metals from Constrained Random-Phase Approximation, Phys. Rev. B 83, 121101(R) (2011).

[189] R. O. Jones and O. Gunnarsson, The Density Functional Formalism, Its Applications and Prospects, Rev. Mod. Phys. 61, 689 (1989).

[190] D. C. Patton, D. V. Porezag, and M. R. Pederson, Simplified Generalized-Gradient Approximation and Anharmonicity: Benchmark Calculations on Molecules, Phys. Rev. B 55, 7454 (1997).

[191] B. Hammer, L. B. Hansen, and J. K. Nørskov, Improved Adsorption Energetics within Density-Functional Theory Using Revised Perdew-Burke-Ernzerhof Functionals, Phys. Rev. B 59, 7413 (1999).

[192] L. Wang, T. Maxisch, and G. Ceder, Oxidation Energies of Transition Metal Oxides within the GGA + U Framework, Phys. Rev. B 73, 195107 (2006).

[193] P. Liu, J. He, B. Kim, S. Khmelevskyi, A. Toschi, G. Kresse, and C. Franchini, Comparative Ab Initio Study of the Structural, Electronic, Magnetic, and Dynamical Properties of $\mathrm{LiOsO}_{3}$ and $\mathrm{NaOsO}_{3}$, Phys. Rev. Mater. 4, 045001 (2020).

[194] L. Kleinman, Density Functional for Noncollinear Magnetic Systems, Phys. Rev. B 59, 3314 (1999).

[195] K. Capelle, G. Vignale, and B. L. Györffy, Spin Currents and Spin Dynamics in Time-Dependent DensityFunctional Theory, Phys. Rev. Lett. 87, 206403 (2001).

[196] M. I. Katsnelson and V. P. Antropov, Spin Angular Gradient Approximation in the Density Functional Theory, Phys. Rev. B 67, 140406(R) (2003).

[197] S. Sharma, J. K. Dewhurst, C. Ambrosch-Draxl, S. Kurth, N. Helbig, S. Pittalis, S. Shallcross, L. Nordström, and E. K. U. Gross, First-Principles Approach to Noncollinear Magnetism: Towards Spin Dynamics, Phys. Rev. Lett. 98, 196405 (2007). 
[198] G. Scalmani and M. J. Frisch, A New Approach to Noncollinear Spin Density Functional Theory beyond the Local Density Approximation, J. Chem. Theory Comput. 8, 2193 (2012).

[199] I. W. Bulik, G. Scalmani, M. J. Frisch, and G. E. Scuseria, Noncollinear Density Functional Theory Having Proper Invariance and Local Torque Properties, Phys. Rev. B 87, 035117 (2013).

[200] F. G. Eich and E. K. U. Gross, Transverse Spin-Gradient Functional for Noncollinear Spin-Density-Functional Theory, Phys. Rev. Lett. 111, 156401 (2013).

[201] F. G. Eich, S. Pittalis, and G. Vignale, Transverse and Longitudinal Gradients of the Spin Magnetization in SpinDensity-Functional Theory, Phys. Rev. B 88, 245102 (2013).

[202] S. Pittalis, G. Vignale, and F. G. Eich, U(1) $\times \mathrm{SU}(2)$ Gauge Invariance Made Simple for Density Functional Approximations, Phys. Rev. B 96, 035141 (2017).

[203] J. J. Goings, F. Egidi, and X. Li, Current Development of Noncollinear Electronic Structure Theory, Int. J. Quantum Chem. 118, e25398 (2018).

[204] C. A. Ullrich, Density-Functional Theory for Systems with Noncollinear Spin: Orbital-Dependent Exchange-Correlation Functionals and Their Application to the Hubbard Dimer, Phys. Rev. B 98, 035140 (2018).

[205] C. A. Ullrich, (Spin-)Density-Functional Theory for OpenShell Systems: Exact Magnetization Density Functional for the Half-Filled Hubbard Trimer, Phys. Rev. A 100, 012516 (2019).

[206] E. A. Pluhar III and C. A. Ullrich, Exchange-Correlation Magnetic Fields in Spin-Density-Functional Theory, Phys. Rev. B 100, 125135 (2019).

[207] D. Hobbs, G. Kresse, and J. Hafner, Fully Unconstrained Noncollinear Magnetism within the Projector Augmented-Wave Method, Phys. Rev. B 62, 11556 (2000).

[208] G. Bergerhoff, R. Hundt, R. Sievers, and I. D. Brown, The Inorganic Crystal Structure Database, J. Chem. Inf. Model. 23, 66 (1983).

[209] P. Villars, N. Onodera, and S. Iwata, The Linus Pauling File (LPF) and Its Application to Materials Design, J. Alloys Compd. 279, 1 (1998).

[210] P. S. White, J. R. Rodgers, and Y. Le Page, CRYSTMET: A Database of the Structures and Powder Patterns of Metals and Intermetallics, Acta Crystallogr. Sect. B 58, 343 (2002).

[211] P. Villars and K. Cenzual, Pearsons Crystal Data: Crystal Structure Database for Inorganic Compounds, ASM International/Material Phases Data System, Vitznau, Switzerland (2010), https://crystalimpact.com/pcd/ Default.htm.

[212] SpringerMaterials: The Landolt-Börnstein database (2020), https://libraries.usc.edu/databases/springer-materialslandolt-bornstein.

[213] C. Bale, E. Bélisle, P. Chartrand, S. Decterov, G. Eriksson, K. Hack, I.-H. Jung, Y.-B. Kang, J. Melançon, A. Pelton, C. Robelin, and S. Petersen, FactSage Thermochemical Software and Databases-Recent Developments, CALPHAD: Comput. Coupling Phase Diagrams Thermochem. 33, 295 (2009).
[214] P. Linstrom and W. Mallard, NIST Chemistry WebBook, NIST Standard Reference Database No. 69 (National Institute of Standards and Technology, Gaithersburg, MD, 2015), https://doi.org/10.18434/T4D303.

[215] MatWeb: Material Property Data, Database of Materials Data Sheets (2020), http://www.matweb.com.

[216] MatNavi: NIMS Materials Database (2014), https://mits .nims.go.jp/en/.

[217] H. Okamoto, P. Villars, and A. Prince, Handbook of Ternary Alloy Phase Diagrams (ASM international, Materials Park, OH, 1995), pp. 10378-10379.

[218] G. Hautier, C. Fischer, V. Ehrlacher, A. Jain, and G. Ceder, Data Mined Ionic Substitutions for the Discovery of New Compounds, Inorg. Chem 50, 656 (2011).

[219] R. F. Berger and J. B. Neaton, Computational Design of Low-Band-Gap Double Perovskites, Phys. Rev. B 86, 165211 (2012).

[220] L. Cheng, R. S. Assary, X. Qu, A. Jain, S. P. Ong, N. N. Rajput, K. Persson, and L. A. Curtiss, Accelerating Electrolyte Discovery for Energy Storage with HighThroughput Screening, J. Phys. Chem. Lett. 6, 283 (2015).

[221] J. Carrete, N. Mingo, S. Wang, and S. Curtarolo, Nanograined Half-Heusler Semiconductors as Advanced Thermoelectrics: An Ab Initio High-Throughput Statistical Study, Adv. Funct. Mater. 24, 7427 (2014).

[222] H. Zhu, G. Hautier, U. Aydemir, Z. M. Gibbs, G. Li, S. Bajaj, J.-H. Pöhls, D. Broberg, W. Chen, A. Jain, M. A. White, M. Asta, G. J. Snyder, K. Persson, and G. Ceder, Computational and Experimental Investigation of $\mathrm{TmAgTe}_{2}$ and $X Y Z_{2}$ Compounds, A New Group of Thermoelectric Materials Identified by First-Principles High-Throughput Screening, J. Mater. Chem. C 3, 10554 (2015).

[223] M. T. Dunstan, A. Jain, W. Liu, S. P. Ong, T. Liu, J. Lee, K. A. Persson, S. A. Scott, J. S. Dennis, and C. P. Grey, Large Scale Computational Screening and Experimental Discovery of Novel Materials for High Temperature $\mathrm{CO}_{2}$ Capture, Energy Environ. Sci. 9, 1346 (2016).

[224] W. Chen, J.-H. Pö, G. Hautier, D. Broberg, S. Bajaj, U. Aydemir, Z. M. Gibbs, H. Zhu, M. Asta, G. J. Snyder, B. Meredig, M. A. White, K. Persson, and A. Jain, Understanding Thermoelectric Properties from High-Throughput Calculations: Trends, Insights, and Comparisons with Experiment, J. Mater. Chem. C 4, 4414 (2016).

[225] I. Petousis, D. Mrdjenovich, E. Ballouz, M. Liu, D. Winston, W. Chen, T. Graf, T. D. Schladt, K. A. Persson, and F. B. Prinz, High-Throughput Screening of Inorganic Compounds for the Discovery of Novel Dielectric and Optical Materials, Sci. Data 4, 160134 (2017).

[226] J. Dagdelen, J. Montoya, M. de Jong, and K. Persson, Computational Prediction of New Auxetic Materials, Nat. Commun. 8, 323 (2017).

[227] Y. Umeda, H. Hayashi, H. Moriwake, and I. Tanaka, Materials Informatics for Dielectric Materials, and Jpn, J. Appl. Phys. 57, 11UB01 (2018).

[228] A. M. Tehrani, A. O. Oliynyk, M. Parry, Z. Rizvi, S. Couper, F. Lin, L. Miyagi, T. D. Sparks, and J. Brgoch, Machine Learning Directed Search for Ultraincompressible, Superhard Materials, J. Am. Chem. Soc. 140, 9844 (2018). 
[229] R. Chen, H. C. Po, J. B. Neaton, and A. Vishwanath, Topological Materials Discovery Using Electron Filling Constraints, Nat. Phys. 14, 55 (2018).

[230] J. A. Flores-Livas, L. Boeri, A. Sanna, G. Profeta, R. Arita, and M. Eremets, A Perspective on Conventional High-Temperature Superconductors at High Pressure: Methods and Materials, Phys. Rep. 856, 1 (2020).

[231] A. I. Liechtenstein, M. Katsnelson, V. Antropov, and V. Gubanov, Local Spin Density Functional Approach to the Theory of Exchange Interactions in
Ferromagnetic Metals and Alloys, J. Magn. Magn. Mater. 67, 65 (1987).

[232] L. Udvardi, L. Szunyogh, K. Palotás, and P. Weinberger, First-Principles Relativistic Study of Spin Waves in Thin Magnetic Films, Phys. Rev. B 68, 104436 (2003).

[233] I. Turek, J. Kudrnovský, V. Drchal, and P. Bruno, Exchange Interactions, Spin Waves, and Transition Temperatures in Itinerant Magnets, Philos. Mag. 86, 1713 (2006). 